\title{
MATHEMATICAL MODELS FOR DYNAMICS OF MOLECULAR PROCESSES IN LIVING BIOLOGICAL CELLS A SINGLE PARTICLE TRACKING APPROACH
}

\author{
AleKsander Weron
}

The X Annual Lecture dedicated to the memory of Professor Andrzej Lasota

\begin{abstract}
In this survey paper we present a systematic methodology of how to identify origins of fractional dynamics. We consider three models leading to it, namely fractional Brownian motion (FBM), fractional Lévy stable motion (FLSM) and autoregressive fractionally integrated moving average (ARFIMA) process. The discrete-time ARFIMA process is stationary, and when aggregated, in the limit, it converges to either FBM or FLSM. In this sense it generalizes both models. We discuss three experimental data sets related to some molecular biology problems described by single particle tracking. They are successfully resolved by means of the universal ARFIMA time series model with various noises. Even if the finer details of the estimation procedures are case specific, we hope that the suggested checklist will still have been of great use as a practical guide. In Appendices $\mathrm{A}-\mathrm{F}$ we describe useful fractional dynamics identification and validation methods.
\end{abstract}

Received: 02.03.2017. Accepted: 26.10.201\%. Published online: 17.05.2018.

(2010) Mathematics Subject Classification: 60J70, 62M10, 92C37.

Key words and phrases: stochastic processes, fractional particle dynamics, ARFIMA time series, identification and validation algorithms.

Research supported by NCN-DFG Beethoven Grant No.2016/23/G/ST1/04083. 


\section{Introduction}

According to E. P. Wigner - the Nobel Prize winner in Physics 1963 It is important to point out that the mathematical formulation of the physicists often crude experience leads in an uncanny number of cases to an amazingly accurate description of a large class of phenomena ([80]).

The Nobel Prize in Physiology or Medicine 2009 was awarded for the discovery of how chromosomes are protected by telomeres and the enzyme telomerase. The Nobel Prize in Chemistry 2012 was given for studies of Gprotein-coupled receptors. And the Nobel Prize in Chemistry 2014 was presented for the development of superresolved fluorescence microscopy. Definitely, the research behind these three Nobel Prizes - awarded within a short five year period - have caused a dramatic increase of experimental and theoretical achievements in the study of living cells around the world. Ultimately the accessibility of quantitative data prompted many physicists and applied mathematicians to turn their attention to the study of single biological cells and the physiological processes running off therein. In the spirit of the above citation of Eugene Wigner, this involvement of physicists and mathematicians has led to a new level of understanding of biological systems. Very recently, the creation of Quantitative Biology sections in the life sciences, hiring physicists and mathematicians in their departments, provides a good mirror of this development.

In this context let us recall that probably Professor Hugo Steinhaus was a first Polish mathematician working actively on specific problems of biology and medicine. He published a series of articles on: garniture pattern of leucocytes ([25]) or arrangement of chromosomes in human cells, on distribution of telomeres, centromeres and orientation of chromosomes in the metaphase (45] and [46]). In a similar spirit Professor Andrzej Lasota, as a versatile mathematician, was interested in the application of existing mathematical tools to biological and medical problems. A spectacular example is his series of articles on a mathematical model of blood cell reproduction and scientific cooperation with Professor Maria Ważewska-Czyżewska who applied those results in the treatment of certain types of drug-induced anemia ([50]).

A phenomenon observed in recent single-molecule experiments is anomalous diffusion, which largely departs from the classical Brownian diffusion theory since the mean-squared displacement (MSD) is nonlinear. The most popular theoretical models that are commonly employed are: continuous-time random walk (CTRW) ([62]), obstructed diffusion (OD) ([33, 65]), fractional Brownian motion (FBM) ([32, 10]), fractional Lévy $\alpha$-stable motion (FLSM) ([15]), fractional Langevin equation (FLE) ([44]) and autoregressive fractionally integrated moving average (ARFIMA) (see [14] and references therein). For the latter process, we note that the acronyms "ARFIMA" and "FARIMA" 
are often used interchangeably in the literature. Yet another popular anomalous diffusion models have to be mentioned, for example those related to fluorescence recovery after photobleaching (FRAP): time-dependent diffusion coefficient model $([66,70])$ and scaled Brownian motion ([51, 27]).

Traditionally, fractional dynamics is related to the concept of fractional dynamic equations. This is an active field of study in physics, mechanics, mathematics, and economics, investigating the behaviour of objects and systems that are described by using differentiation of fractional orders. The celebrated fractional Fokker-Planck equation (FFPE) corresponding to the popular CTRW model, describing anomalous diffusion in the presence of an external potential $V(x)$, is given by the following formula

$$
\frac{\partial w(x, t)}{\partial t}={ }_{0} D_{t}^{1-\alpha}\left[\frac{\partial}{\partial x} \frac{V^{\prime}(x)}{\eta}+K \frac{\partial^{2}}{\partial x^{2}}\right] w(x, t) .
$$

It was derived explicitly in [61, 1], where methods of its solution were introduced and for some special cases exact solutions were calculated. Here, the operator ${ }_{0} D_{t}^{1-\alpha}, \alpha \in(0,1)$, is the fractional derivative of the RiemannLiouville type $([64])$. It is known that ${ }_{0} D_{t}^{1-\alpha}$ introduces a convolution integral with a slowly decaying power-law kernel, which is typical for memory effects in complex systems ([71]). In (1.1), $w(x, t)$ denotes the probability density function (PDF) and the prime (') stands for the derivative with respect to the space coordinates relating the force $F(x)$ and the potential through $F(x)=-V^{\prime}(x)$. The constant $K$ denotes the anomalous diffusion coefficient, whereas $\eta$ is the generalized friction constant. For $\alpha \rightarrow 1,(1.1)$ becomes the ordinary Fokker-Planck equation. The FFPE describes subdiffusion in accordance with the mean-squared displacement in the force-free limit and it obeys some generalized fluctuation-dissipation theorem. Moreover, a generalization of the Einstein-Stokes-Smoluchowski relation $K=k_{B} T / \eta$ connects the generalized friction and diffusion coefficients ([62]).

Derivatives and integrals of fractional orders are used to describe objects that can be characterized by long (power-like) memory or self-similarity. In recent years, there has been a great interest in long-range dependent and selfsimilar processes, in particular FBM, FLSM and ARFIMA. As a candidate suitable for extensive statistical analysis of the fractional dynamics we choose here the ARFIMA model $([31,5,3])$. It is a discrete time analog of the overdamped fractional Langevin equation ([55]) that allows for the non-Gaussian law (Lévy $\alpha$-stable) and the long memory.

The objective of this work is to convince the readers that the ARFIMA process can serve as a universal and simple discrete time model for fractional dynamics of empirical data and the celebrated FBM and FLSM form the limiting case of ARFIMA. Moreover, since the ARFIMA models were successful 
in analyzing data in other fields (econometrics, see 2003 Nobel Prize in Economic Sciences for C.W.J. Granger and R. Engel; finance and engineering in [23, 26, 29]), many statistical tools (and computer packages, e.g. ITMS in [5]) are available and are presented in Appendix D. We also provide a basic checklist for convenience of the inexperienced user as to what statistical techniques must be applied in various stages of the analysis of the empirical data.

The paper is structured as follows. In section 2 three models for fractional dynamical systems are considered, namely FBM, FLSM and ARFIMA time series. The former two models are self-similar and their increments form long-range dependent processes. The latter discrete-time ARFIMA process is stationary, and when aggregated, in the limit, it converges to either FBM or FLSM. In this sense it generalizes both models. In contrast to them, it allows for different light- and heavy-tailed distributions, and, as a stationary process, provides tools for calculating predictions. In section 3 we discuss three data sets related to the molecular biology problems. They are successfully resolved by means of the universal ARFIMA time series model with various noises. Even if the finer details of the estimation procedures are case specific, we hope that the suggested checklist will still have been of great use as a practical guide.

In Appendices $\mathrm{A}-\mathrm{F}$ we describe useful fractional dynamics identification and validation methods. We present an estimation algorithm for the ARFIMA parameters in the case of noise belonging to the domain of attraction of the stable law for both positive and negative memory parameters. We also present methods of testing of stationarity and ergodicity, distribution type, self-similarity and long memory. In particular we present two efficient methods of estimation self-similarity index and memory parameter with the help of $p$-variation and sample mean-squared displacement respectively.

\section{Basic models for fractional dynamics}

In this section we discuss three models for fractional dynamics: fractional Brownian motion (section 2.1), fractional Lévy stable motion (section 2.2), and ARFIMA (section 2.3). The former two models are self-similar and their increments form long-range dependent processes. The discrete-time ARFIMA process is stationary and generalizes both models since aggregated, in the limit, it converges to either fractional Brownian or Lévy stable motion. In contrast to them, it allows for different light- and heavy-tailed distributions, and both long (power-like) and short (exponential) dependencies ([14]). Moreover, as a stationary process, it provides prediction tools. 


\subsection{Fractional Brownian motion}

Fractional Brownian motion introduced by Kolmogorov in 1940 ([43, [58]) is a generalization of the classical Brownian motion (BM). Most of its statistical properties are characterized by the self-similarity (Hurst) exponent $0<H<1$. For any $0<H<1$, fractional Brownian motion (FBM) of index $H$ (Hurst exponent) is the mean-zero Gaussian process $B_{H}(t)$ with the following integral representation $([58])$ :

$$
B_{H}(t)=\int_{-\infty}^{\infty}\left\{(t-u)_{+}^{H-\frac{1}{2}}-(-u)_{+}^{H-\frac{1}{2}}\right\} d B(u),
$$

where $B(t)$ is a standard Brownian motion and $(x)_{+}=\max (x, 0)$.

FBM is $H$-self-similar, namely for every $c>0$ we have $B_{H}(c t) \stackrel{D}{=} c^{H} B_{H}(t)$ in the sense of all finite dimensional distributions, and has stationary increments. It is the only Gaussian process satisfying these properties. With probability 1 , the graph of $B_{H}(t)$ has both Hausdorff dimension and box dimension of $2-H$.

For $H>1 / 2$, the increments of the process are positively correlated and exhibit positive long-range dependence, whereas for $H<1 / 2$, the increments of the process are negatively correlated and exhibit negative long-range dependence (see Appendix C). For the second moment of the FBM we have $<B_{H}^{2}(t)>=\sigma^{2} t^{2 H}$, where $\sigma>0$.

The precise simulation of such process is of great interest. The most commonly used approaches can be split in two categories. The first one, related to theoretically exact methods, has so far been composed only of a matrix factorization technique based on the Cholesky decomposition of the covariance matrix. Unfortunately, this technique has a complexity of $O\left(N^{2}\right)$ and requires high computational resources even for moderate trajectory lengths. The other category is composed of nonexact techniques. All of the above methods have their particular drawbacks and advantages. The choice between them boils down to a tradeoff between speed and accuracy. However, Davies and Harte in 24] proposed both fast and exact synthesis method for stationary Gaussian processes, called the circulant embedding method. It can be easily applied for a FBM ([18]). Since based on the fast Fourier transform (FFT) algorithm, its complexity is only $O(N \log N)$. 


\subsection{Fractional Lévy stable motion}

FBM can be generalized to a fractional Lévy stable motion (FLSM) ([38, 75]). FLSM is the $\alpha$-stable process $L_{H}^{\alpha}(t)$ with the following integral representation:

$$
L_{H}^{\alpha}(t)=\int_{-\infty}^{\infty}\left\{(t-u)_{+}^{d}-(-u)_{+}^{d}\right\} d L_{\alpha}(u)
$$

where $L_{\alpha}(t)$ is an $\alpha$-stable motion (SM), $0<\alpha \leq 2,0<H<1$, and $d=$ $H-1 / \alpha$.

The process for $\alpha=2$ becomes a FBM, is $H$-self-similar and has stationary increments. Analogously to the FBM case, we say the increments of the process exhibit positive long-range dependence if $d>0(H>1 / \alpha)$, and negative dependence when $d<0(H<1 / \alpha)([74])$. This is due to the behaviour of the integrand (kernel function) in 2.1).

When $d<0$, the kernel function has singularities at $u=0$ and $u=t$. These singularities magnify the stable noise process $d L_{\alpha}(t)$, cause large spikes in the paths of the FLSM. Their dependence structure resembles that of a negatively correlated process and thus we shall refer to this case as to the negative dependence scenario. In the case when $d>0$ the kernel is bounded and positive, for all $t>0$. Thus the jumps in the paths of $L_{H}^{\alpha}(t)$ due to the fluctuations of the noise process are not as magnified as in the case $d<0$. In this case especially for large values of $H$, the kernel function decays slowly as $u \rightarrow-\infty$. This implies that the past fluctuations in the process $d L_{\alpha}(t)$ influence significantly the present values of the process $L_{H}^{\alpha}(t)$. This case is referred to as the positive long-range dependence scenario. Therefore, as in the Gaussian case, the parameter $d$ controls sign of dependence.

Simulation of such process is even more difficult than in the FBM case. This is due to the fact that stable processes cannot be characterized by twodimensional distributions, as in the Gaussian case. Namely, any Gaussian random vector is fully defined by the mean and covariance matrix, whereas stable vectors are defined in terms of so-called spectral measure ([38]), which is a much more complicated object. Hence, there are no exact methods of simulation of FLSM in the literature. The most commonly used approaches apply integral representation of FLSM. Stoev and Taqqu in 74] proposed a fast method based on the FFT algorithm with complexity only $O(N \log N)$. It can be also applied to ARFIMA processes and more general moving average stable processes. 


\subsection{ARFIMA process}

Autoregressive fractionally integrated moving average (ARFIMA) time series were introduced by Granger and Joyeux ([31]) and Hosking ([37]). The ARFIMA process $\left\{X_{t}\right\}$, denoted by $\operatorname{ARFIMA}(p, d, q)$, is defined by:

$$
\Phi_{p}(B) X_{t}=\Psi_{q}(B)(1-B)^{-d} Z_{t},
$$

where innovations $Z_{t}$ are i.i.d. random variables with either finite or infinite variance. $\Phi_{p}(z)=1-\phi_{1} z-\phi_{2} z^{2}-\ldots-\phi_{p} z^{p}$ is the autoregressive polynomial and $\Psi_{q}(z)=1+\psi_{1} z+\psi_{2} z^{2}+\ldots+\psi_{q} z^{q}$ is the moving average polynomial. The operator $(1-B)^{-d}$ is an integrating operator and has infinite binomial expansion

$$
(1-B)^{-d} Z_{t}=\sum_{j=0}^{\infty} b_{j}(d) Z_{t-j},
$$

where the $b_{j}(d)^{\prime}$ s are coefficients in the expansion of the function $f(z)=$ $(1-z)^{-d},|z|<1$, i.e.

$$
b_{j}(d)=\frac{\Gamma(j+d)}{\Gamma(d) \Gamma(j+1)}, \quad j=0,1, \ldots,
$$

where $\Gamma$ is the gamma function.

The sequence $\left\{Z_{t}\right\}$ is often called a "noise process (sequence)" ([5]). We assume that innovations $Z_{t}$ are i.i.d. and belong to the domain of attraction of an $\alpha$-stable law with $0<\alpha \leq 2$. The series given by $(2.3)$ is convergent almost surely (a.s.) and ARFIMA definition 2.2 is correct if and only if

$$
\alpha(d-1)<-1 \quad \Longleftrightarrow d<1-\frac{1}{\alpha} .
$$

In particular, in the Gaussian case $(\alpha=2)$ we have $d<1 / 2$. Moreover, if all roots of the polynomial $\Phi_{p}$ lie outside the unit circle, the $\operatorname{ARFIMA}(p, d, q)$ time series defined by 2.2 is stationary and has a causal moving average form

$$
X_{t}=\sum_{j=0}^{\infty} c_{j}(d) Z_{t-j}
$$

(for details see [37, 41]). Stationary ARFIMA processes are also ergodic ([74]). In this paper we concentrate on such a case. 
Such processes are asymptotically $H$-self-similar with the parameter $H=$ $d+1 / \alpha$. Precisely, we have $N^{-H} \sum_{k=1}^{[N t]} X_{k} \rightarrow L_{H}^{\alpha}(t)$ for $t \geq 0$ and the convergence is with respect to finite-dimensional distributions ([74]). Hence the ARFIMA process can be considered as a discrete-time generalization of FLSM (in particular of FBM).

In the Gaussian case, the rate of decay of the autocovariance function of the ARFIMA model is

$$
r(k)=<X(0) X(k)>\sim k^{2 d-1}, \quad \text { as } k \rightarrow \infty,
$$

where $<X(0) X(k)>$ denotes the mean value of $X(0) X(k)$. Therefore, for $d>0$ we have $\sum_{k=0}^{\infty}|r(k)|=\infty$. This serves as a classical definition of long memory and is equivalent to the case of the increments of FBM with $H>1 / 2$. Similarly, for $d<0$ we arrive at the negative short (but power-like) dependence, which corresponds to the increments of FBM with $H<1 / 2$. The case $d=0$ leads to the autoregressive moving average (ARMA) model, which has exponentially decaying autocorrelation function $([5,69])$.

Finally, the linear predictor for the ARFIMA process based on the finite past $X_{n}, \ldots, X_{0}$ takes the form:

$$
\hat{X}_{n+h}=\sum_{j=0}^{n} a_{j} X_{n-j},
$$

where the sequence $\left\{a_{0}, a_{1}, \ldots, a_{n}\right\}$ is given by $a_{j}=-\sum_{t=0}^{k-1} c_{t} h_{j+k-t}$, the $c_{j}$ 's are defined by (2.4) and $h_{j}$ 's are given by

$$
\frac{\Phi_{p}(z)(1-z)^{d}}{\Theta_{q}(z)}=\sum_{j=0}^{\infty} h_{j} z^{j}, \quad|z|<1,
$$

see [40] for the discussion of the prediction problem in the infinite variance ARFIMA case.

Simulation of ARFIMA processes is by no means straightforward. For such processes, except for the special case of $\operatorname{ARMA}(p, q)$ model, there are no exact methods. The existing algorithms concentrate mostly on efficient ways to calculate the infinite sum given in (2.4). Stoev and Taqqu (74]) proposed a fast method based on the FFT algorithm with complexity only $O(N \log N)$. It can be also applied to FLSM and more general moving average stable processes.

The $\operatorname{ARFIMA}(0, d, q)$ is called $\operatorname{FIMA}(d, q)$ and is represented by the following equation

$$
(1-B)^{d} X(t)=Z(t)-\psi_{1} Z(t-1)-\ldots-\psi_{q} Z(t-q),
$$


where $t=0, \pm 1, \ldots, B$ is a shift operator: $B X(t)=X(t-1),-1 / 2<d<1 / 2$, which takes fractional values, either positive or negative, and $\{Z(t)\}$ is a white noise sequence $([5])$.

In many applications $\operatorname{ARFIMA}(1, d, 1)$ model is sufficient to describe the data well, see, e.g. [12, 14]. The basic building blocks of $\operatorname{ARFIMA}(1, d, 1)$ model are $\mathrm{AR}(1)$ process: $X(t)=\phi_{1} X(t-1)+Z(t)$, and MA(1) process: $X(t)=Z(t)-\psi_{1} Z(t-1)$. AR(1) is a causal or future-independent function of noise and stands for the regression. The explanatory variable is the observation immediately prior to our current observation. In order to get an idea about the role of MA part let us concentrate on the case when $Z(t)$ is a white noise. It appears that if $X(t)$ is a stationary 1-correlated time series, i.e. $X(s)$ and $X(t)$ are independent whenever $|t-s|>1$ (in contrast to an i.i.d. sequence which is zero-dependent), then it can be represented as the MA(1) process ([5]). The dependence (correlation in the white noise case) is only one lag long and its intensity is fully controlled by the parameter $\psi_{1}$. Hence, ARMA models introduce short memory of the process. In general, the $\mathrm{MA}(q)$ process may reconstruct an arbitrary short time (finite lag) correlation structure from the experimental data. The fractional integration introduces the long (power-law) memory, which is defined by the memory parameter $d$.

Its special case, $\operatorname{FIMA}(d, 1)$ is given by the following formula:

$$
(1-B)^{d} X(t)=Z(t)-\psi Z(t-1),
$$

where $t=0, \pm 1, \ldots$.

Brownian motion (BM) corresponds, in the limit sense (see [74]), to $\operatorname{FIMA}(0,0)$. Similarly, FBM corresponds to $\operatorname{FIMA}(d, 0)$ with $d=H-1 / 2$, where $H$ is the self-similarity parameter. Hence, it is possible to model and characterize more complex processes than with FBM alone. The FIMA processes offer flexibility in modelling long power-law and one-lag dependencies by choosing the memory parameter $d$ and the appropriate moving average coefficient $\psi$ in 2.5 .

We will illustrate how the methodology proposed in the Appendix can be applied to the verification of the ergodic property using simulated trajectories of the following two processes:

(i) ergodic (increments) of fractional Brownian motion (fBm):

$$
X(t)=\int_{-\infty}^{\infty}\left\{(t-u)_{+}^{H-\frac{1}{2}}-(-u)_{+}^{H-\frac{1}{2}}\right\} d B(u)
$$

where $B(t)$ is a Brownian motion,

(ii) non-ergodic subordinated Ornstein-Uhlenbeck process (sOU):

$$
X(t)=Z\left(S_{\alpha}(t)\right)
$$


where $Z(t)$ is an Ornstein-Uhlenbeck process

$$
d Z(t)=-\lambda Z(t) d t+\sigma d B(t)
$$

and $S_{\alpha(t)}$ is the inverse $\alpha$-stable subordinator independent of $Z(t)$ defined in the following way:

$$
S_{\alpha}(t)=\inf \left\{\tau>0: U_{\alpha}(\tau)>t\right\}
$$

where $U_{\alpha}(t)$ is the $\alpha$-stable subordinator with Laplace transform given by $e^{-t u^{\alpha}}$. In the simulations we use the following parameters: $H=0.4, \alpha=0.8$, $\lambda=0.5, \sigma=0.1$. Moreover, we assume that $X(0)=0$ for $\mathrm{fBm}$ and $X(0) \sim$ $N\left(0, \frac{\sigma^{2}}{2 \lambda}\right)$ for sOU. Sample trajectories of the analyzed processes and their increments are plotted in Figs. 1 and 2 .
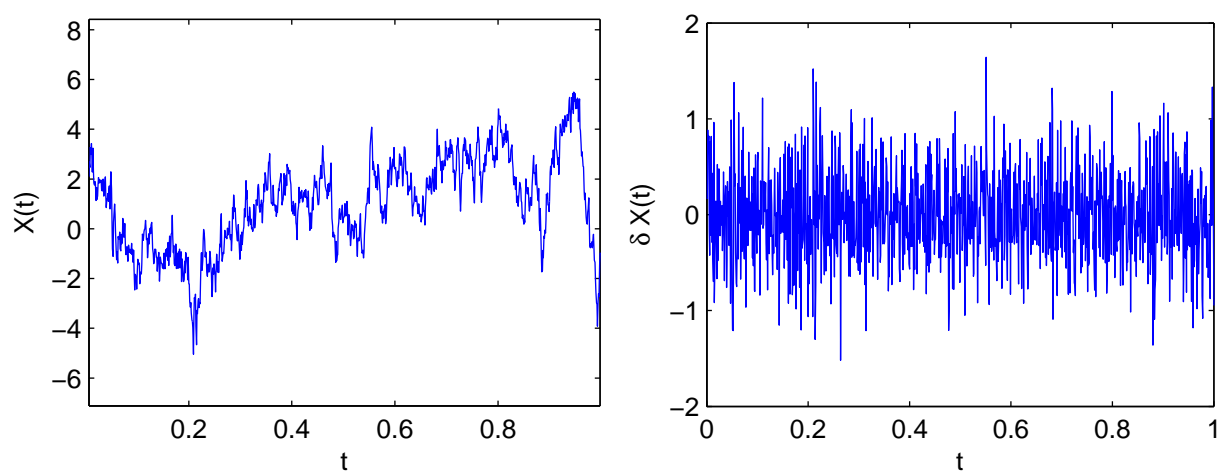

Figure 1. Sample trajectory (left hand panel) and it's increments (right hand panel) of the fractional Brownian motion with $H=0.4$, source: [53]
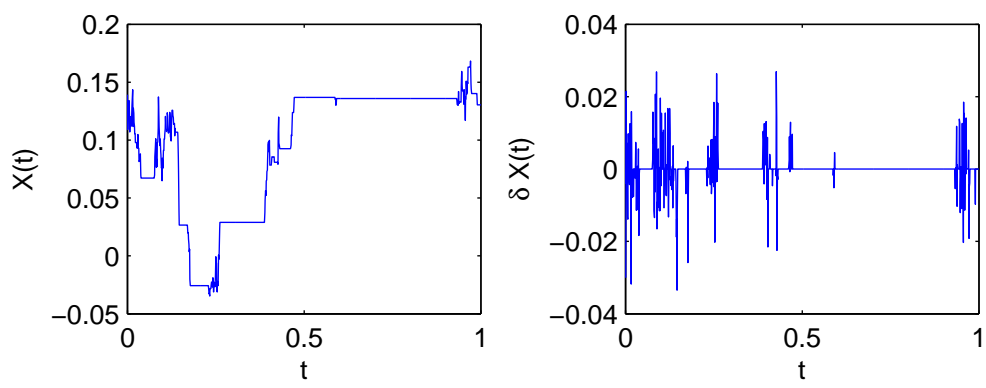

Figure 2. Sample trajectory (left hand panel) and it's increments (right hand panel) of the subordinated Ornstein-Uhlenbeck process with $\alpha=0.8, \lambda=0.5, \sigma=0.1$, source: 53 . 
One should remember that ergodicity is well defined only for stationary processes. Hence, stationarity of the process distribution is the first property to check before proceeding to ergodicity analysis. A standard way is to plot quantile lines ([38]).

Next, for both of the analysed sample paths we calculate the empirical dynamical functional. Note that since we analyse single trajectories the ensemble average in the definition of theoretical $D(n)$, see Appendix $\mathrm{B}$.

$$
D(n) \approx \widehat{D}(n)=\frac{1}{N-n+1} \sum_{k=0}^{N-n} \exp \{i[X(n+k)-X(k)]\}
$$

is replaced by using the empirical $\widehat{D}(n)$. The results are plotted in Figures 3 and 4 for the classical Ornstein-Uhlenbeck process (OU) and for the subordinated Ornstein-Uhlenbeck process (sOU), respectively. What can be
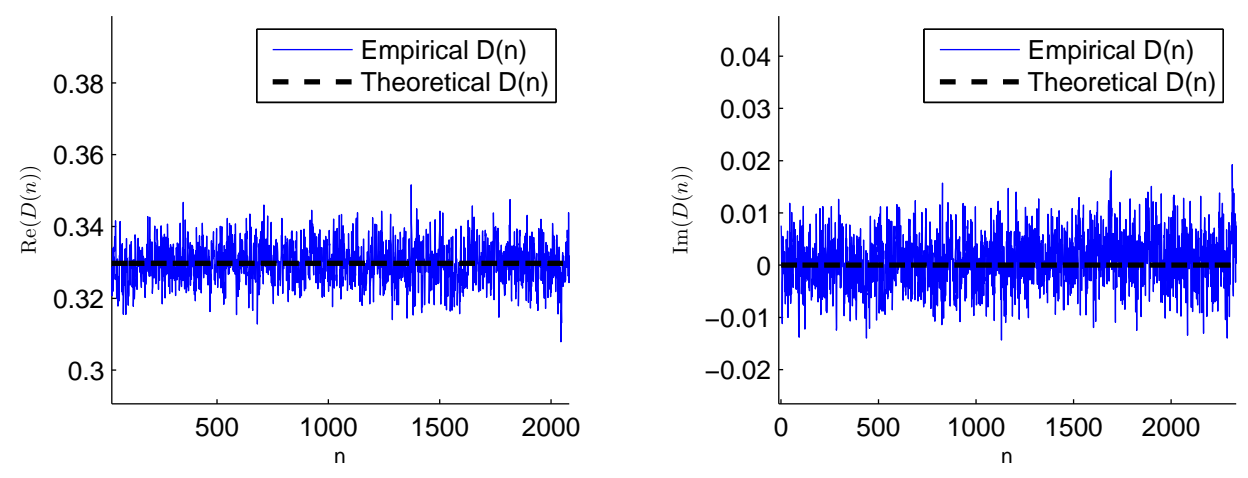

Figure 3. Empirical $D(n)$ of the Ornstein-Uhlenbeck process (blue solid line) as well as theoretical $D(n)$ obtained from formula in [53] (dashed black line). Note that in the left hand panel the real part of $D(n)$ is plotted while in the right hand panel the imaginary part, source: [53]

easily noticed from these pictures is that, while for the OU process the dynamical functional converges rapidly to some constant, for the sOU process there is no convergence. Such result might indicate on ergodicity of OU process and clearly proves non-ergodicity of the sOU process. Further, we use the analytical formulas obtained in the paper. We estimate parameters of $\operatorname{ARFIMA}(1,0,0)$ process and calculate the theoretical asymptotic value of that model. We obtain $\phi=0.31$ for OU process and $\phi=0.45$ for the sOU process. The theoretical dynamical functionals for the analyzed cases are plotted in Figures 3 and 4 together with the corresponding empirical values. What we can observe is that for the OU process these values coincide, which confirms that the analyzed sample trajectory indeed comes from the ARFIMA model with autoregression property and no memory. Obviously, such model 

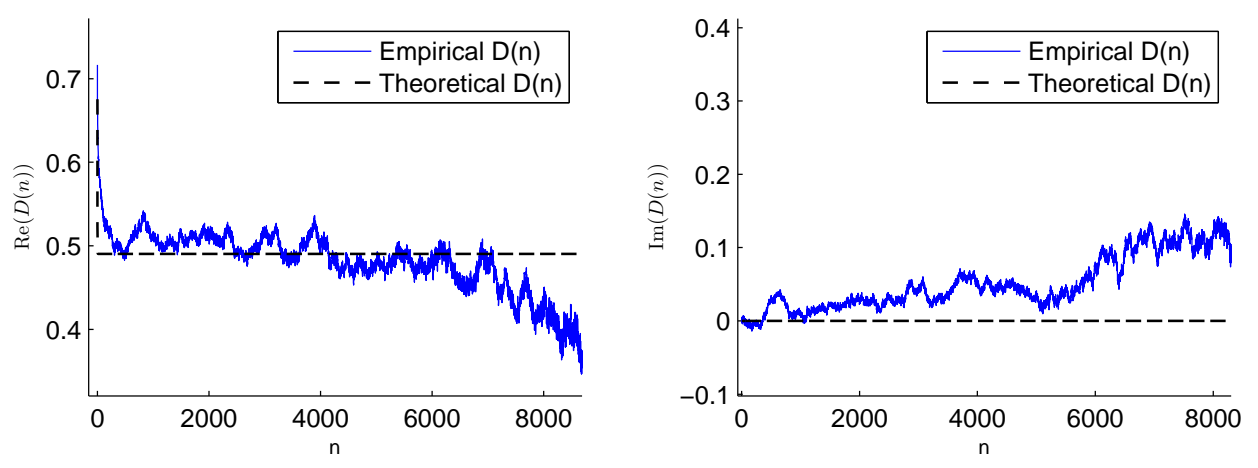

Figure 4. Empirical $D(n)$ of the subordinated Ornstein-Uhlenbeck process (blue solid line) as well as theoretical $D(n)$ obtained from formula in 53 . (dashed black line). Note that in the left hand panel the real part of $D(n)$ is plotted while in the right hand panel the imaginary part, source: [53]

is ergodic. On the other hand, for the sOU process the obtained values are different even in the long time limit. As a consequence, the structure of the analyzed trajectory is significantly different from an ergodic ARFIMA model. This simple example illustrates in what way, using the results derived in the paper, we can verify if the analyzed data comes from a huge general class of ARF IMA models and conclude on it's ergodicity.

\section{Modelling of fractional dynamics}

In this section we illustrate the applicability of ARFIMA processes to three different kinds of biological SPT data. We will study the data by applying the following general checklist proposed in [16] for testing fractional dynamics.

CheCKList. Testing for fractional dynamics

L1 Stationarity and ergodicity (prerequisites for ARFIMA modelling) (see Appendices $A(B)$

L2 Sample MSD (to check for the presence of the power-law dependence and anomalous diffusion) (see Appendix (C)

L3 Fitting the ARFIMA parameters (usually it is enough to fit at most $\operatorname{ARFIMA}(1, d, 1)$, so three parameters are to be estimated) (see Appendix D)

L4 Fitting the distribution to the noise (Gaussian, non-Gaussian stable, other) (see Appendix E) 
L5 (optional) H-self-similarity for the partial sum process (along with the concept of surrogate data it provides a double check for both the memory parameter $d$ and stability index $\alpha$ ) (see Appendix F)

Anomalous diffusion in crowded fluids, e.g. in cytoplasm of living cells, is a frequent phenomenon ([36]). Crowded fluids form a wide class of soft condensed matter systems. Prominent examples provide dense polymer solutions and the cytoplasm of living cells (34).

The motion of tracer particles may switch from normal to anomalous diffusion, i.e. the tracers's mean square displacement (MSD) exhibits a sublinear growth with time $\operatorname{MSD}(t) \sim t^{a}$, where $a<1$ for pure anomalous subdiffusion. In the transient anomalous subdiffusion one mechanism operates at short times and another at long times, depending on a crossover time. Despite the frequent observation of such a behavior in crowded fluids, the origin of the anomaly remains controversial ([67]).

Why does anomalous subdiffusion matter? According to [67], first, it affects reaction kinetics through the time dependent diffusion coefficient. Second, it is a probe of submicroscopic organization, though unfortunately far from uniquely invertible. Moreover, ubiquitous observation in cell biology is that the diffusive motion of macromolecules and organelles is anomalous, see [36].

The progress in understanding the molecular nature of intracellular processes in the last years is astonishing, and is due to tight collaboration of physicists, chemists, biologists and mathematicians, all working in the same direction and using specific methods of their sciences ([70, 11]).

\subsection{Fitting ARFIMA to the mRNA data}

As a universal candidate suitable for extensive statistical analysis of the subdiffusive dynamics in biological cells we propose here the ARFIMA model which was described in the previous section. We apply the introduced methodology to the single mRNA molecule time series from the Golding and Cox microscopy video ([30, 12]). The analyzed video has 1801 frames of size $59 \times 76$ pixels, which present location of mRNA molecule at the time $t_{k}=k \mathrm{sec}$, for $k=1, \ldots, 1801$. The center of pixel in the upper-left and bottom-right corner of each frame has coordinates $(1,1)$ and $(59,76)$, respectively. We identified the position of a molecule at the time $t_{k}$ with the position of its mass center $\left(\bar{x}\left(t_{k}\right), \bar{y}\left(t_{k}\right)\right)$, which is calculated based on image segmentation results obtained by application of the approach introduced in [20].

Consequently, we obtained a $2 \mathrm{D}$ data set of $\left(\bar{x}\left(t_{k}\right), \bar{y}\left(t_{k}\right)\right), k=1, \ldots, 1801$. These data form time series of a single mRNA molecule in the analyzed microscopy video $([12])$. We analyze a $2 \mathrm{D}$ trajectory $\left\{\left(X_{n}, Y_{n}\right)=\left(\bar{x}\left(t_{n}\right), \bar{y}\left(t_{n}\right)\right)\right.$ : $n=1, \ldots, 1801\}$ and concentrate on the increments of $x$ and $y$ coordinates. 
All analysis is based on two sets of increments $\left\{X_{n+1}-X_{n}: n=1, \ldots, 1800\right\}$ and $\left\{Y_{n+1}-Y_{n}: n=1, \ldots, 1800\right\}$ of coordinates $x$ and $y$, respectively.

Step L1. Trajectories and their increments of both coordinates of the data are presented in Figure 5. A simple examination of the presented increments
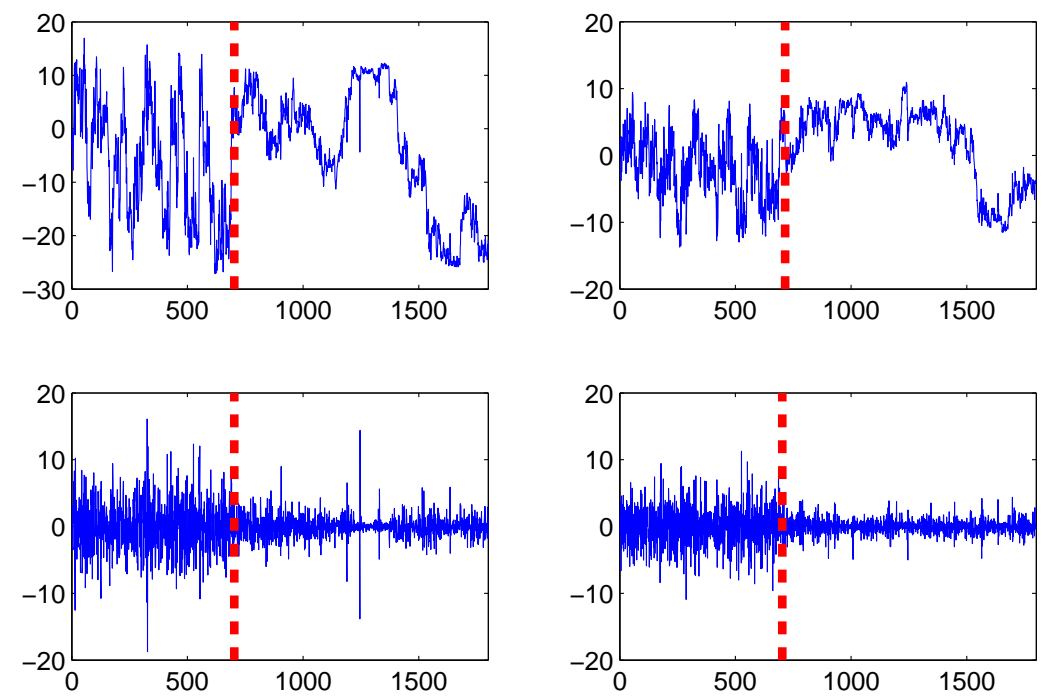

Figure 5. A plot of the trajectories (top panels) and their increments (bottom panels) with a change point in variance (red dashed line), for $x$ (left hand panels) and $y$ coordinates (right hand panels). The variance changing points are 702th and 715th observations for $x$ and $y$ coordinates, respectively, source: [12]

tells us that the data are not stationary in general. They demonstrate two different regimes. Hence, by using the variance change point test ([28]) we split the data for two stationary subsets. The changing points are 702 th and 715 th observation for $x$ and $y$ coordinates, respectively. In sum, we obtain four subsets which we denote by $X 1, X 2, Y 1$ and $Y 2$.

Next, we applied the ergodicity test (see Appendix B) to all four subsets of the data to check the necessary condition for ergodicity $(\overrightarrow{B .6})$, which requires only one appropriately long trajectory. In Figure 7 we see the result of the test for the X1 and X2 subsets. The necessary condition for mixing (B.5) is clearly satisfied. This implies that ergodicity cannot be rejected. We also checked that all subsets satisfy necessary conditions for mixing (B.5).

Step L2. In order to check the power-law dependence and subdiffusive behavior of the data we calculated the sample MSD (see Appendix C). First, we calculated sample MSD for all four subsets of the data: $X 1, X 2, Y 1$ and 

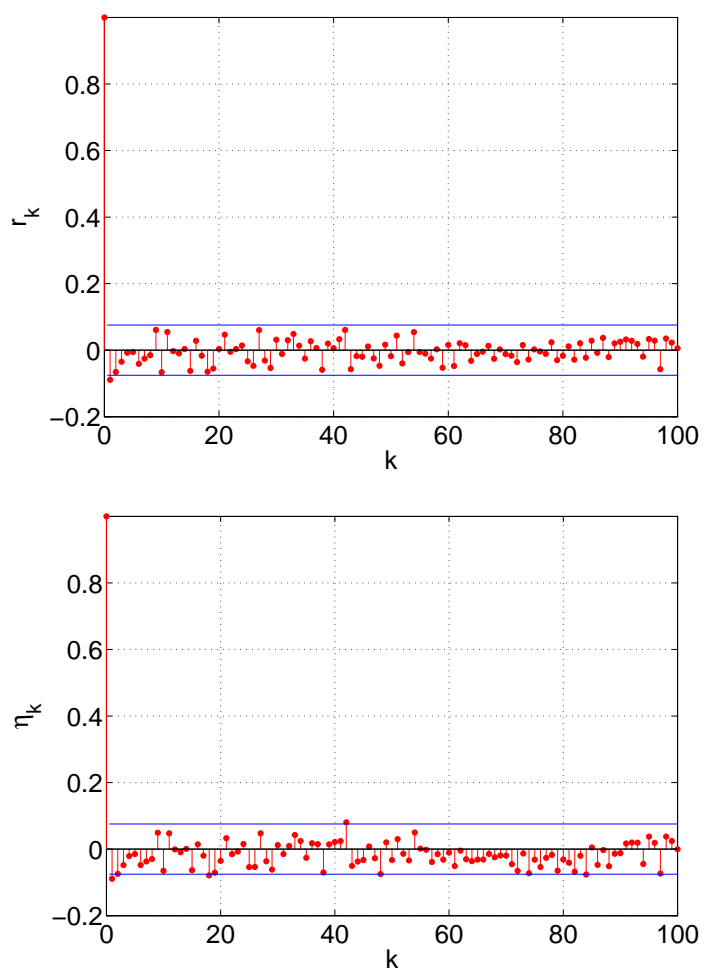

Figure 6. Autocorrelation (top panel) and partial autocorrelation (bottom panel) functions corresponding to the subset X1 of data. Both functions tend to zero indicating stationarity of the sample. Moreover, all values of the functions lie between $95 \%$ confidence bounds calculated for the white noise case which suggests the $\operatorname{ARFIMA}(0, d, 0)$ model, source: [16]

$Y 2$ and obtained the following $d$ estimates: $-0.1,-0.19,-0.28,-0.22$, respectively. Next, we did it for the 2D data: $(X 1, Y 1),(X 2, Y 2)$ and $(X=X 1 \cup X 2$, $Y=Y 1 \cup Y 2$ ), where the distance was calculated according to Euclidean norm. The results were: $-0.15,-0.20,-0.16$, respectively. We can see that in all cases $d$ is essentially negative (the negative power-law dependence case), hence the process clearly follows the subdiffusive dynamics.

Step L3. We fitted the $\operatorname{ARFIMA}(1, d, 1)$ model (see Appendix D) to the four time series ([12]). The best-fitted ARFIMA models were: $\operatorname{ARFIMA}(0,-0.13,0)$, $\operatorname{ARFIMA}(0,-0.14,1)(\psi=0.13), \operatorname{ARFIMA}(1,-0.07,1)(\phi=0.48, \psi=0.67)$ and ARFIMA $(0,-0.38,0)$. The orders of the model were chosen looking at the autocorrelation and partial autocorrelation functions (see Appendix A) and 

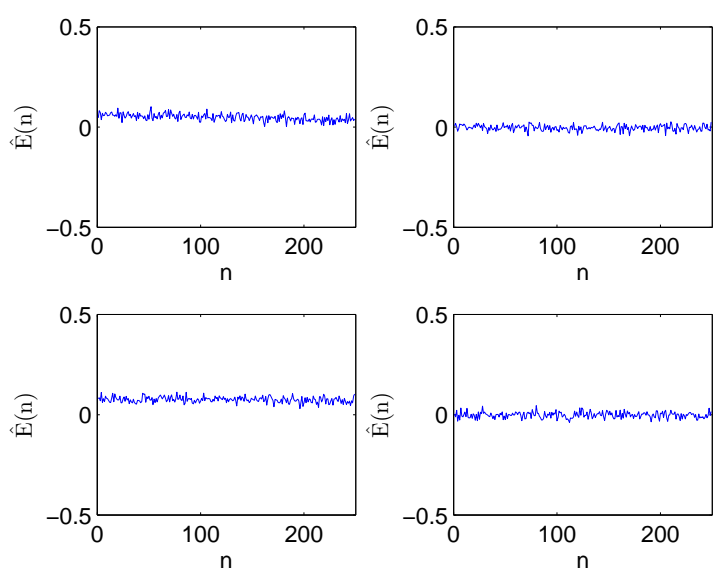

Figure 7. Real (left hand panels) and imaginary (right hand panels) parts of the function $\hat{E}(n)$ corresponding to the subsets X1 (top panels) and X2 (bottom panels) of the data, source: [16. The necessary condition for mixing B.5 is clearly satisfied. This implies that ergodicity cannot be rejected

checking the independence of the noise. The noise was calculated by means of ITSM package ([5]). The independence for the above models could not be rejected for the Ljung-Box, turning points, difference-sign and rank tests (for the information about the tests, see, e.g., [5, 8, 7]).

Step L4. Next, we fitted different distributions to the noise, namely Gaussian, Lévy stable and normal-inverse Gaussian (NIG). A random variable $X$ is said to have a NIG distribution if it has density

$$
f(x)=\frac{\alpha \delta}{\pi} e^{\delta \sqrt{\alpha^{2}-\beta^{2}}+\beta(x-\mu)} \frac{\mathrm{K}_{1}\left(\alpha \sqrt{\delta^{2}+(x-\mu)^{2}}\right)}{\sqrt{\delta^{2}+(x-\mu)^{2}}}, \quad-\infty<x<\infty .
$$

The NIG distribution, introduced in [2], is described by four parameters $(\alpha, \beta, \delta, \mu)$, where $\alpha$ stands for tail heaviness, $\beta$ for asymmetry, $\delta$ is the scale parameter, and $\mu$ is the location. The normalizing constant $K_{\lambda}(t)$ in 3.1 is the modified Bessel function of the third kind with index $\lambda$, also known as the MacDonald function.

The NIG law is the only one that cannot be rejected for any part of the data (see [12]). To confirm the goodness of fit of the model, we also calculated sample MSD for 1000 simulated trajectories of ARFIMA models with the fitted parameters given and compared the results with the MSD values obtained for the original data. We can see in Figure 8 that the fitted ARFIMA processes reproduce the sample MSD well. 


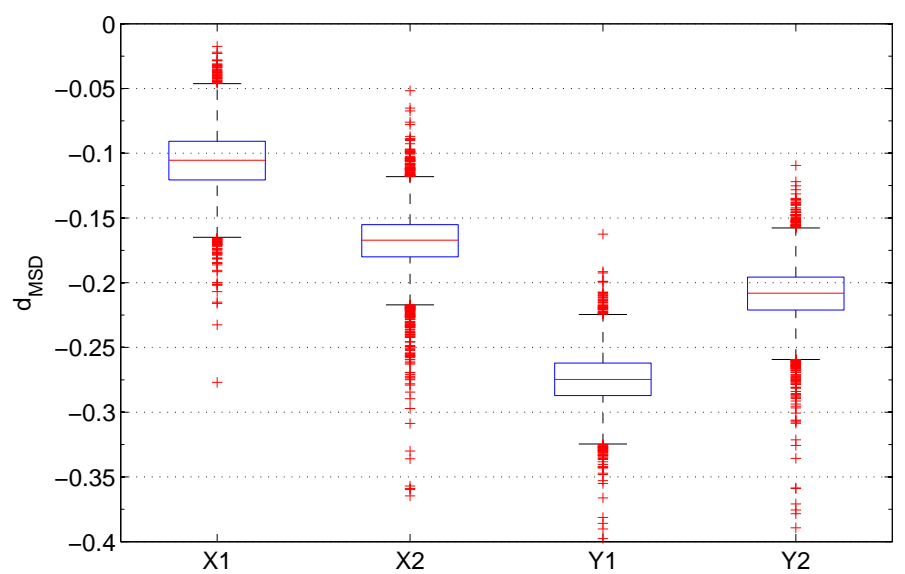

Figure 8. Boxplots of sample MSD calculated for 10000 simulated trajectories of the ARFIMA processes fitted to the four subsets of the analyzed data. We can observe that the ARFIMA model leads to the MSD values which are close to those from the empirical data, source: [7]

The proposed ARFIMA model is universal, nevertheless, it turns out, that the four stationary subsets of the 2D trajectory have different parameters $\left(\phi_{1}, d, \psi_{1}\right)$. This is due to the fact that our single particle tracking analysis is not a mean-value approach as it was done in [30]. Observe that the shape of the cells and crowded fluid characteristic of the cytoplasm influence the dynamics of the labelled mRNA molecules. In particular, we believe that the parameters of the fitted ARFIMA models can provide some insight into the physical reasons for subdiffusive motion of the molecule. Namely, the parameters $d$ in both directions $x$ and $y$ are influenced by the shape of the cell. Simulations show that, e.g., as the width of the biological cell gets smaller then the memory parameter becomes "more negative". Two additional parameters $\phi_{1}$ and $\psi_{1}$ which are responsible for short-time effects are influenced by short-distance interactions in a crowded fluid environment in the cytoplasm ([12]).

Step L5. Finally, we studied the surrogate data of the four subsets. From the results for the surrogate data (see Appendix E), the corresponding estimates for the parameter $1 / \alpha$ were: $1 / \alpha_{p \text { Var }}=H_{p V a r}=0.53,0.5,0.47,0.5$, respectively, where $H_{p V a r}$ is the self-similarity estimator obtained via the $p$-variation method (see Appendix F). We observe that the values confirm that the distributions of the noises belong to the domain of attraction of the Gaussian $(\alpha=2)$ law.

Recall that both the self-similarity index $H$ and type of distribution $1 / \alpha$ have impact on $d$, since $d=H-1 / \alpha$. 


\subsection{Dynamical functional for telomeres data from the U2OS cancer cell line}

We start with an analysis of a single trajectory of fluorescently labeled telomeres in the nucleus of living human cells originating from the U2OS cancer cell line (for a detailed data description see [10]). The analysed trajectory is plotted in Figure 9. Since the considered ARFIMA model is a stationary process, we will consider the increments of the $X$ coordinate, i.e. $d X\left(t_{n}\right)=X\left(t_{n+1}\right)-X\left(t_{n}\right)$, where $t_{1}, t_{2}, \ldots, t_{N}$ are the time points of the measurements. A trajectory of the process $d X$ is plotted in the bottom panel of Figure 9 .
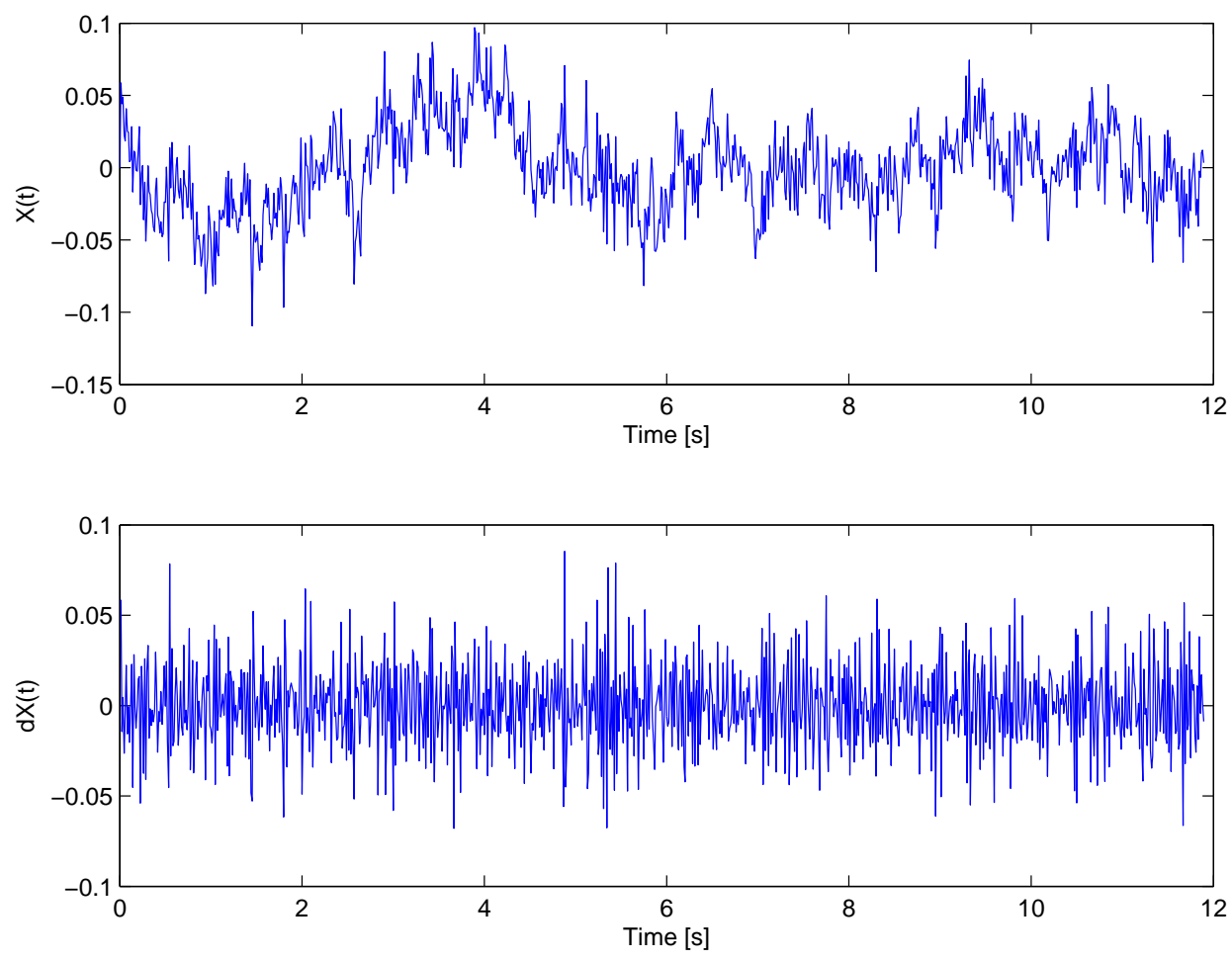

Figure 9. X coordinate of telomere trajectory (top panel) and it's increments (bottom panel)

In paper [10] the authors showed that the telomere motion is driven by a fractional Gaussian noise with negative memory parameter. Recall that $\operatorname{ARFIMA}(0, d, 0)$ is a discrete version of the fractional noise process. Moreover, it was recently shown in [9] that $\operatorname{ARFIMA}(0, d, 1)$ process can be an appropriate model for anomalous diffusion data with measurement errors and 
the MA part can help in the identification of these errors in such experiments. Being motivated by the mentioned results and the fact that experimental data usually contains some measurement errors, we fit a Gaussian $\operatorname{ARFIMA}(0, d, 1)$ model to the $d X$ process of the analysed trajectory. A detailed guideline for the ARFIMA model parameters estimation one can find e.g. in [16]. We obtain the following parameter values $d=-0.3138$, $\theta=-0.6536$ and $\sigma=0.0199$. Next, we test for the Gaussianity of the noise process. To this end, we apply a Kolmogorov-Smirnov goodness-of-fit test and obtain $p$-value $=0.44$. Because the obtained $p$-value is well above the standard significance level of $5 \%$, the Gaussianity hypothesis can not be rejected and with high certainty we can assume that it is true.

Having estimated the $A R F I M A(0, d, 1)$ model parameters we can use the theoretical findings of $[53$ and calculate the values of the dynamical functional $D(n)$ for such model. At the same time we can calculate the empirical value of $D(n)$ for the analysed trajectory. Note that since we focus on only one trajectory we use the time average instead of the ensemble one. The results are plotted in Figure 10. The obtained curves almost coincide, both for the
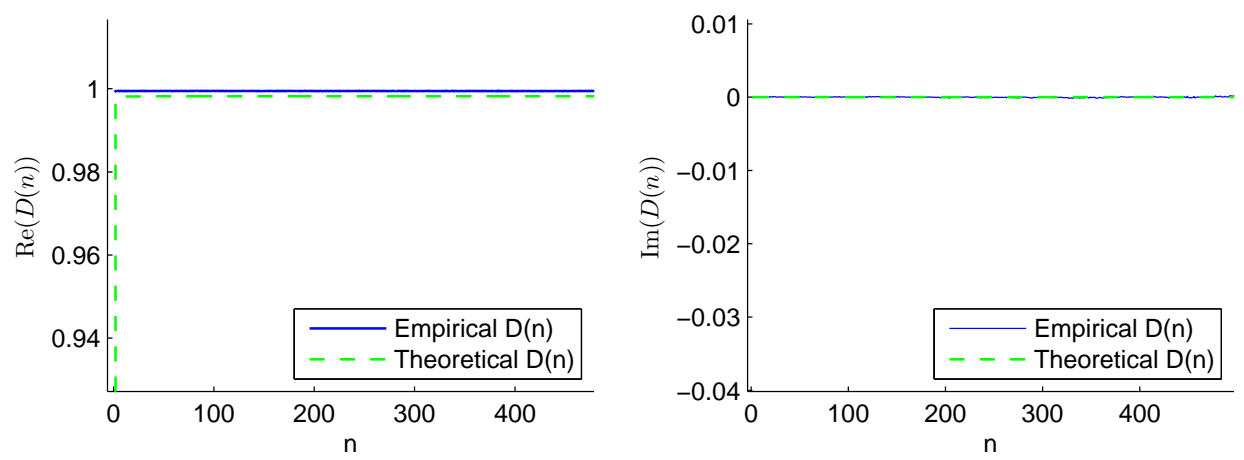

Figure 10. $D(n)$ of Gaussian $\operatorname{ARFIMA}(0, d, 1)$ fitted to telomere data (dashed green line) and empirical $D(n)$ calculated from increments of a single telomere trajectory (solid blue line). The real part of $D(n)$ is plotted in the left hand panel, while the imaginary part in the right hand panel, source: [53]

real part (left hand panel) and the imaginary part (right hand panel), which shows similar behaviour of the telomere data as for the theoretical model. Hence, we can confirm that the telomere motion can be described by the ARFIMA $(0,-0.32,1)$ model. Recall that such a model is characterized by a negative memory responsible for subdiffusion. Moreover, it has no autoregression part, so the lengths of the telomere moves are not linearly dependent on the previous steps. Further, the $M A(1)$ part is found in the data, which means that there is a correlation between the error terms of the consecutive telomere position recordings. Such a property can be related to some recurrent measurement errors. Finally, since the $\operatorname{ARFIMA}(0,-0.32,1)$ model is 
ergodic and we have rigorously confirmed that it's theoretical behaviour of the dynamical functional resembles the empirical behaviour of the dynamical functional for telomere increments, we can conclude that the telomere motion is ergodic.

\subsection{Dynamical functional for mRNA molecules inside live $E$. coli cells}

Now we turn to the second example, illustrating behaviour of the dynamical functional for experimental data, namely movements of mRNA molecules inside live E. coli cells recorded by Golding and Cox ([30]) in SPT experiment. We focus on a single trajectory, which is the longest one out of the whole dataset. Analogously as in the previous example, we analyse the incremental process $d X$. The mRNA trajectory is plotted in the top panel of Figure 11, while the process $d X$ is plotted in the bottom panel.
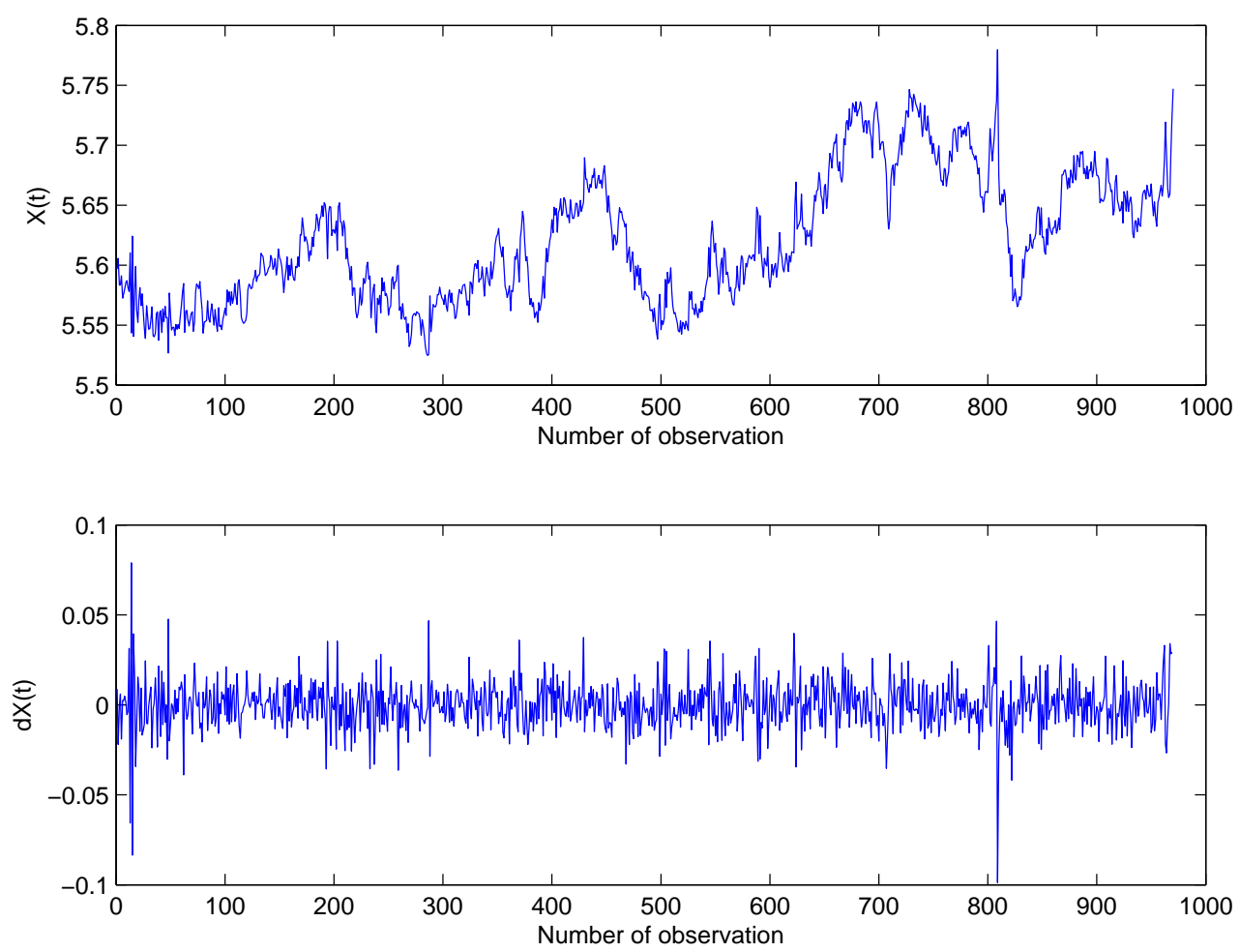

Figure 11. mRNA trajectory (top panel) and it's increments (bottom panel) 
The same trajectory was analysed in [7] and the author showed that the $\operatorname{ARFIMA}(1, d, 1)$ model with $\alpha$-stable noise process can be used to model the increments of the mRNA motion. We use the same model parameters as estimated in the mentioned work, i.e. $d=-0.16, \phi=-0.02$ and $\theta=0.12$. Next, we fit a symmetric $\alpha$-stable distribution for the noise process. We obtain $\alpha=1.86$ and $\sigma=0.0083$. Note that the remaining $\beta$ and $\mu$ parameters are equal to 0 . In order to confirm that the chosen distribution fits the analysed data, we perform the Kolmogorov-Smirnov goodness-of-fit test. The obtained $p$-value is equal 0.78 and is well above the standard $5 \%$ significance level, so we can assume that the $\alpha$-stable distribution is appropriate for modelling the mRNA data.

Further, we compare the analytical formula for the dynamical functional from [53] with the estimated model parameters and the dynamical functional calculated from mRNA data. Again, since we analyse a single trajectory, ensemble average is replaced with time average. The obtained results are plotted in Figure 12 .
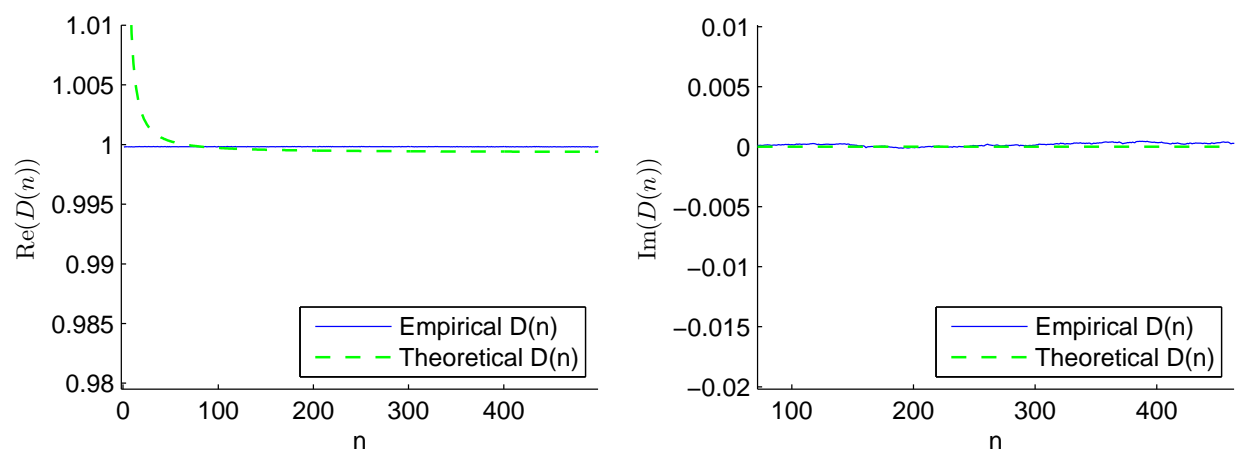

Figure 12. $D(n)$ of $\alpha$-stable $A R F I M A(1, d, 1)$ fitted to mRNA data (dashed green line) and empirical $D(n)$ calculated from increments of a single mRNA trajectory (solid blue line). The real part of $D(n)$ is plotted in the left hand panel, while the imaginary part in the right hand panel, source: [53]

As we can observe, the empirical values of $D(n)$ almost coincide with the theoretical functional form for both, the real (left hand panel of Figure 12) and the imaginary part (right hand panel of Figure 12). Hence, we have obtained another confirmation that the $\alpha$-stable $\operatorname{ARFIMA}(1,-0.16,1)$ model can be used to describe the mRNA motion in live $E$. coli cells. This model is ergodic and we have shown that its theoretical behaviour of the dynamical functional resembles the empirical behaviour of the dynamical functional for mRNA motion increments. As a consequence, we can conclude that the mRNA motion is also ergodic. The model parameters indicate on subdiffusive behaviour of the data (negative memory parameter $d$ ), autoregression of order one, i.e linear 
dependence between the values in the consecutive time steps $(A R(1)$ part) and the correlation between error terms $(M A(1)$ part).

The last two examples illustrate how the functional form of $D(n)$ can be used for the verification if the chosen ARFIMA model fits the data and what conclusions does it yield on the properties of the underlying phenomenon. A possibility to verify ergodic properties of a process, and hence use of the Boltzmann hypothesis, is an essential issue for studies of the real-life phenomena. One of the statistical tools that might be utilized in this context is the dynamical functional $D(n)$.

Further, using examples of simulated Ornstein-Uhleneck processes of two types (i.e. ergodic and non-ergodic) and biophysical data, we have illustrated that the obtained analytical formula for $D(n)$ can be used to verify if a chosen model fits the experimental data, i.e. if the empirical and theoretical values coincide and what conclusions does it yield on the properties of the underlying phenomenon. Since the $\alpha$-stable ARFIMA model is a discrete time version of many celebrated fractional processes (like e.g. fractional Brownian motion or fractional Levy motion), the obtained results yield a convenient tool that may help in an analysis of anomalous diffusion dynamics.

\section{Appendix A. Stationarity}

All tools presented here assume that the data are either stationary or increment stationary ([11]). Stationary and non-stationary processes are very different in their properties, and they require different inference procedures. First, note that a simple and useful method to tell if a process is stationary in empirical studies is to plot the data. Loosely speaking, if a series does seem to have a linear trend, seasonal trend or a varying volatility, then very likely, it is not stationary. In this case one has to transform the data to make them stationary. To this end, it is usually enough to remove the trends by means of fitting and subtracting a polynomial and a trigonometric function.

For a more refined diagnostic of dependence and stationarity, autocorrelation function $(\mathrm{ACF})$ and partial autocorrelation function (PACF) are examined. The sample autocorrelation function at lag $k$ is defined as

$$
r_{k}=\frac{\sum_{t=1}^{N-k}\left(X_{t}-\bar{X}\right)\left(X_{t+k}-\bar{X}\right)}{\sum_{t=1}^{N}\left(X_{t}-\bar{X}\right)}, \text { for } k=0,1, \ldots, K
$$


and stands for a measure of the linear dependence between observations with time lag $k$ ([5]). The partial autocorrelation function $\eta_{k}$, which, at lag $k$, is defined as a correlation between the predictor errors of values $X_{n}$ and $X_{n+k}$ represented in terms of $X_{n+1}, \ldots, X_{n+k-1}$ ([5]). For any ergodic process, both functions, ACF and PACF, should approach to zero as the lag tends to infinity. They also provide information about the order of the underlying ARMA model. This leads to a simple visual test for stationarity.

Algorithm 1. Stationarity test for one sample ([16])

(i) Plot the data. If the data posses a clear linear or seasonal trend, try to remove it.

(ii) Calculate $A C F$ and PACF. If they converge to zero, this suggests stationarity of the underlying process. In particular:

(a) For i.i.d. data the autocorrelation function should be zero for all $k \neq 0$. The partial autocorrelation function should be equal to zero except for the lag $k=0$.

(b) For moving average processes of order $q(M A(q))$, the autocorrelation function should be zero for lags beyond $q$. Hence, it can be used for estimating the $q$ parameter.

(c) For autoregressive processes of order $p(A R(p))$, the partial autocorrelation function should be zero for lags beyond $p$. Hence, it can be used for estimating the $q$ parameter.

We also note that if the model possesses both the MA and MA parts then the reasoning about the orders of the models (estimation of $p$ and $q$ ) can be quite misleading ([5]).

If a number of realizations of a phenomena is recorded, the stationarity property of the analyzed datasets can be checked by means of so-called quantile lines. Let us assume that we observe $M$ samples of length $N$ and denote their values by $\left\{Z_{n}^{k}\right\}, n=1,2, \ldots N, k=1, \ldots, M$, and $0<p_{j}<1, j=1, \ldots, J$ are given probabilities.

Algorithm 2. Stationarity test for many samples ([77])

(i) Derive estimators of the corresponding quantiles $q_{j}(n)=F_{n}^{-1}\left(p_{j}\right)$, where $F_{n}=F_{n}(x)$ denotes $C D F$ of the random variable $Z_{n}$ represented by the statistical sample $Z_{n}^{k}, k=1, \ldots, M$.

(ii) Obtain the approximation of the so-called quantile lines, i.e. the curves $q_{j}=q_{j}(n)$ defined by the condition $P\left\{Z_{n} \leq q_{j}(n)\right\}=p_{j}$. In layman terms, the quantile lines represent the value $q_{j}$ for which $p_{j} * 100 \%$ of the data are below at a certain time point $n$.

(iii) For a stationary process the quantile lines $q_{j}(n)=$ const, whereas for a self-similar process they behave like $n^{H}$. 


\section{Appendix B. Mixing and ergodicity}

We describe now another two fundamental properties of the data: ergodicity and mixing. Ergodicity of the stationary process $Y(n)$ means that its phase space cannot be divided into two nontrivial sets such that a point starting in one set will never get to the second set. Let us emphasize that for every stationary and ergodic process the Boltzmann ergodic hypothesis, enabling better analysis of the data characteristics, is satisfied, i.e. the temporal and ensemble averages coincide.

Another fundamental property is mixing, i.e. the asymptotic independence of the random variables $Y(n)$ and $Y(0)$ as $n$ goes to infinity. It is well-known that mixing is a stronger property than ergodicity (49]). Thus to show ergodicity it is enough to prove mixing, which is easier in many cases.

To this end, we use the dynamical functional (DF) test recently developed in [56] (see also [48]). It is based on a concept of the dynamical functional ([38]). The dynamical functional $D(n)$ corresponding to the process $Y(n)$ is defined as

$$
D(n)=\langle\exp \{i[Y(n)-Y(0)]\}\rangle .
$$

Thus, $D(n)$ is actually a Fourier transform of $Y(n)-Y(0)$ evaluated for the Fourier-space variable $k=1$. The following result illustrates the strength of the dynamical functional ([19]). The stationary ID process $Y(n)$ is mixing if and only if

$$
\lim _{n \rightarrow \infty} D(n)=|\langle\exp \{i Y(0)\}\rangle|^{2} .
$$

The above condition should be viewed as the asymptotic independence of $Y(n)$ and $Y(0)$ as $n \rightarrow \infty$. Moreover, if $Y(n)$ is Gaussian, then the dynamical functional is equal to $D(n)=\exp \left\{\sigma^{2}[r(n)-1]\right\}$, where $r(n)$ is the autocorrelation function of $Y$ and $\sigma^{2}$ is the variance of $Y(0)$. Thus, in the Gaussian case, condition (B.2) is equivalent to the fact that $r(n) \rightarrow 0$ as $n \rightarrow \infty$.

The above condition (B.2) can be written in the equivalent form

$$
\lim _{n \rightarrow \infty} E(n)=0,
$$

where

$$
E(n)=D(n)-|\langle\exp \{i Y(0)\}\rangle|^{2} .
$$

The DF test holds for all infinitely divisible stationary processes ([56]). 
Consequently, using formula $(\mathrm{B} .2)$ we get that the stationary ID process $Y(n)$ is ergodic if and only if

$$
\lim _{n \rightarrow \infty} \frac{1}{n} \sum_{k=0}^{n-1} D(k)=|\langle\exp \{i Y(0)\}\rangle|^{2} .
$$

Equivalently, $Y(n)$ is ergodic if and only if

$$
\lim _{n \rightarrow \infty} \frac{1}{n} \sum_{k=0}^{n-1} E(k)=0 .
$$

It should be mentioned that in order to check necessary and sufficient conditions for mixing $(\overline{B .3}$ ) and ergodicity $(\overline{B .4})$ in the language of dynamical functional (B.1), the reasonable length of each analyzed trajectory should not be shorter than 500 points. The number of trajectories needed to calculate ensemble averages depends strongly on the underlying distribution. For the Gaussian case it is enough to have about 100 trajectories, but it is not enough for a heavy-tailed $\alpha$-stable law. Every distribution needs to be analyzed separately.

It gets much more complicated, when there is not enough trajectories to calculate ensemble averages, see [53, 54]. Suppose that we have only one realization of the process $Y(n), n=0,1, \ldots, N$, where $N$ is an appropriately large integer. If we assume that $Y(n)$ is mixing, then Boltzmann ergodic hypothesis is satisfied - the temporal and ensemble averages coincide.

Algorithm 3. Testing of mixing and ergodicity properties on one trajectory $([56,48])$

(i) Approximate the dynamical functional $D(n)$ by

$$
\widehat{D}(n)=\frac{1}{N-n+1} \sum_{k=0}^{N-n} \exp \{i[Y(n+k)-Y(k)]\} .
$$

(ii) Approximate the ensemble average on the right side of (B.2) by

$$
\widehat{a}=\left|\frac{1}{N+1} \sum_{k=0}^{N} \exp \{i Y(k)\}\right|^{2} .
$$

(iii) If

$$
\widehat{E}(n)=\widehat{D}(n)-\widehat{a} \approx 0
$$


for large $n$, then the process can be mixing. Violation of (B.5) implies that $Y(n)$ does not have the mixing property.

(iv) Check the following condition

$$
\frac{1}{n} \sum_{k=0}^{n-1} \widehat{E}(k) \approx 0
$$

for large $n$. If the condition is satisfied, then the process can be ergodic. Its violation implies ergodicity breaking.

It should be underlined that $\mathrm{B} .5$ and $(\mathrm{B} .6)$ are by no means sufficient for mixing and ergodicity, respectively. This means that having only one trajectory of a random process we can prove lack of mixing or ergodicity. To show that the process is mixing or ergodic, ensemble averages need to be calculated $([10])$.

We note that the non-stationary (and non-ergodic) models like CTRW can be distinguished from the ergodic and stationary models (as exemplified by FBM) by applying tests aimed at checking the stationarity of increments or ergodicity. In [60] a test, based on a fractal structure, was introduced to discriminate between FBM and OD.

\section{Appendix C. Memory parameter estimator based on sample mean-squared displacement}

In the literature different methods of assessing long-range dependence and estimating the memory parameter $d$ have been developed ([3]). It is important to realize what are the assumptions and limitations of various tools and what is the exact output of different estimators. This also refers to self-similarity index estimators. For example, a very well-known RS method, in the general stable case, does not return $H$, which is true only in the Gaussian case, but the value $d+1 / 2$, where $d=H-1 / \alpha$ and $\alpha$ is the index of stability.

The sample mean-squared displacement (MSD) for the general Lévy stable case was calculated in [15]. Let $\left\{X_{i}, i=0, \ldots, N\right\}$ be a sample of length $N+1$. Sample MSD $M_{N}(\tau)$ for lag $\tau$ is defined as

$$
M_{N}(\tau)=\frac{1}{N-\tau+1} \sum_{k=0}^{N-\tau}\left(X_{k+\tau}-X_{k}\right)^{2} .
$$


Note the essential difference between increments of $X_{t}$ in (F.1) and C.1. The latter is a moving window, whereas the former is defined on disjoint subintervals (blocks). The sample MSD is a time average MSD on a finite sample regarded as a function of difference $\tau$ between observations. It is a random variable in contrast to the ensemble average which is deterministic. The following fact describes the behaviour of the sample MSD for the partial sum process of the ARFIMA time series. Let $\left\{Y_{i}, i=0, \ldots, N\right\}$ be an $\operatorname{ARFIMA}(p, d, q)$ time series with $\alpha$-stable noise and $1<\alpha \leq 2$. We define its partial sum process $\left\{X_{k}=\sum_{i=1}^{k} Y_{i}, k=0, \ldots, N\right\}$. Then for large $N / \tau$ :

$$
M_{N}(\tau) \stackrel{D}{\sim} \tau^{2 d+1}
$$

Since the normalized partial sum of the ARFIMA process converges to a FLSM with $H=d+1 / \alpha([74)$, we obtain the following fact ([15]). Let $\left\{X_{i}, i=0, \ldots, N\right\}$ be a FLSM with $0<H<1$ and $1<\alpha \leq 2$. Then, for large $N$,

$$
M_{N}(\tau) \stackrel{D}{\sim} \tau^{2 d+1}
$$

where $d=H-1 / \alpha$.

In particular, for a FBM we obtain the well-known result that $M_{N}(\tau) \sim$ $\tau^{2 H}$, and for both BM and LSM we arrive at the diffusion case, namely $M_{N}(\tau) \sim \tau$ since $d=0$.

As a consequence, we see that the memory parameter $d$ controls the type of anomalous diffusion ([15]). If $d<0(H<1 / \alpha)$, so in the negative dependence case, the process follows the subdiffusive dynamics, if $d>0(H>1 / \alpha)$, the character of the process changes to superdiffusive. Moreover, it appears that $\alpha$-stable processes for $\alpha<2$ can serve both as examples of subdiffusion and superdiffiusion. The subdiffusion pattern arises when the dependence is negative, so possible large positive jumps are quickly compensated by large negative jumps, and on average the process travels shorter distances than the light-tailed Brownian motion.

Now, we introduce a method of estimation of the memory parameter $d$ based on the notion of sample MSD. It can be applied to ARFIMA time series and fractional stable noise for both Gaussian $(\alpha=2)$ and non-Gaussian $(\alpha<2)$ cases.

Algorithm 4. Estimation of the memory parameter based on sample $\operatorname{MSD}([8])$

(i) Calculate $M_{N}(\tau)$ for $\tau=1,2, \ldots, 10$.

(ii) Fit the linear regression line according to $\ln \left(M_{N}(\tau)\right)=\ln (C)+a \ln (\tau)$, $\tau=1,2, \ldots 10$, where $C$ and $a$ are constants.

(iii) The estimated value $\hat{d}=(a-1) / 2$. 
The introduced sample MSD estimator is consistent and works remarkably well, especially for the FBM case. Other analyzed processes need more attention due to approximation errors caused by simulation methods and not exact but limiting results. In general, one may check that the variance of the estimator is very low compared with other well-known methods of estimation of $d$, like, e.g. R/S ([7]).

\section{Appendix D. ARFIMA parameter estimation}

Following [42, 13], we define the $(p+q+1)$-dimensional vector $\boldsymbol{\beta}_{0}=$ $\left(\phi_{1}, \phi_{2}, \ldots, \phi_{p}, \psi_{1}, \psi_{2}, \ldots, \psi_{q}, d\right)$, where $\phi_{1}, \phi_{2}, \ldots, \phi_{p}$ and $\psi_{1}, \psi_{2}, \ldots, \psi_{q}$ are the coefficients of the polynomials $\Phi_{p}$ and $\Psi_{q}$ respectively. The vector $\boldsymbol{\beta}_{0}$ belongs to the parameter space $E=\left\{\boldsymbol{\beta}: \phi_{p}, \psi_{q} \neq 0, \Phi_{p}(z) \Psi_{q}(z) \neq 0\right.$ for $|z| \leq 1, \Phi_{p}, \Psi_{q}$ have no common roots, $\left.d \in(-1 / 2,1-1 / \alpha)\right\}$.

The estimation procedure can be summarized in the following steps.

Algorithm 5. ARFIMA parameter estimation ([13])

(i) Calculate the normalized periodogram by

$$
I_{n}(\lambda)=\left|\sum_{t=1}^{n} X_{t} e^{-i \lambda t}\right|^{2},-\pi \leq \lambda \leq \pi .
$$

(ii) The estimator $\boldsymbol{\beta}_{n}$ of the true parameter vector $\boldsymbol{\beta}_{0}$ is defined as

$$
\boldsymbol{\beta}_{n}=\arg \min _{\boldsymbol{\beta} \in E} \int_{\frac{1}{n}}^{\pi} \frac{I_{n}(\lambda)}{g(\lambda, \boldsymbol{\beta})} d \lambda,
$$

where

$$
g(\lambda, \boldsymbol{\beta})=\left|\frac{\Psi_{q}\left(e^{-i \lambda}, \boldsymbol{\beta}\right)}{\Phi_{p}\left(e^{-i \lambda}, \boldsymbol{\beta}\right)\left(1-e^{-i \lambda}\right) d(\boldsymbol{\beta})}\right|^{2} .
$$

If the orders of the model are small, which is often the case, the estimation procedure simplifies. Let us now concentrate on $\operatorname{ARFIMA}(1, d, 1)$. In such a case the parameter space $E$ has three dimensions, i.e. $\boldsymbol{\beta}=\left(\phi_{1}, \psi_{1}, d\right) \in E$, 
polynomials $\Phi_{p}(z)=1-\phi_{1} z, \Psi_{q}(z)=1+\psi_{1} z$ and the power transfer function has the form

$$
g(\lambda, \boldsymbol{\beta})=\left|\frac{1+\psi_{1} e^{-i \lambda}}{1-\phi_{1} e^{-i \lambda}}\right|^{2} \frac{1}{(2-2 \cos \lambda)^{d}} .
$$

Therefore, the estimator $\tilde{\boldsymbol{\beta}}_{n}$ can be rewritten as

$$
\arg \min _{\boldsymbol{\beta} \in E} \int_{\frac{1}{n}}^{\pi} I_{n}(\lambda)\left[\frac{1-2 \phi_{1} \cos \lambda+\phi_{1}^{2}}{1+2 \psi_{1} \cos \lambda+\psi_{1}^{2}}\right](2-2 \cos \lambda)^{d} d \lambda .
$$

In particular, for the $\operatorname{FIMA}(d, 1)$ model, which is feasible in applications, the estimator is

$$
\arg \min _{\boldsymbol{\beta} \in E} \int_{\frac{1}{n}}^{\pi} I_{n}(\lambda) \frac{(2-2 \cos \lambda)^{d}}{1+2 \psi_{1} \cos \lambda+\psi_{1}^{2}} d \lambda
$$

In order to calculate $\tilde{\boldsymbol{\beta}}_{n}$, we have used fminsearch function implemented in Matlab, which applies the simplex search method of [47].

The above estimation procedure requires information about the order of the model, namely the $p$ and $q$ parameters. In practice, in order to estimate the parameters one can investigate the autocorrelation and partial autocorrelation functions (see Appendix A) or, for the increasing orders of the model, stop the search when the noise becomes close to the white noise sequence. Calculation of the noise can be performed by using standard mathematical packages, like, e.g. ITSM ([5]). Checking its independence can be done via standard tests, like the Ljung-Box ([7]).

Another way to estimate the parameters (including the order) of the ARFIMA model is to find such a set of parameters which minimizes the prediction error (the problem is related to so-called backtesting in statistics) ([72]). The prediction formula for ARFIMA processes was discussed in Section 2.3 . This is one of the most time-consuming fitting procedures but it has an advantage that it is very meaningful to practitioners.

\section{Appendix E. Fitting the distribution to the noise}

We now consider in detail two possible probability laws underlying the noise (and, consequently, the data): Gaussian and $\alpha$-stable. To check if the noise comes from a population with a different distribution, one can perform 
various statistical tests based on the empirical distribution function, e.g. the Kolmogorov-Smirnov and Cramer-von Mises ([6, 7]).

Stable distributions, also called $\alpha$-stable are ubiquitous in nature due to generalized central limit theorem. It says that the stable distributions, like the Gaussian one, attract distributions of sums of independent identically distributed random variables $([38])$. Due to this reason, stable distributions naturally appear when evolution of a system or result of an experiment are determined by a sum of many random factors.

A random variable $X$ is stable if for some $\alpha \in(0 ; 2], \sigma \in(0 ; \infty), \beta \in[-1 ; 1]$, $\mu \in \mathbb{R}$ its characteristic function has the following form ([38]):

$$
\phi_{X}(t)=\left\{\begin{array}{lll}
\exp \left(-\sigma^{\alpha}|t|^{\alpha}\left(1-i \beta \operatorname{sgn}(t) \tan \frac{\pi \alpha}{2}\right)+i t \mu\right) & \text { if } \quad \alpha \neq 1, \\
\exp \left(-\sigma|t|\left(1+i \beta \frac{2}{\pi} \operatorname{sgn}(t) \ln |t|\right)+i t \mu\right) & \text { if } \quad \alpha=1 .
\end{array}\right.
$$

Therefore, each stable distribution is characterized by four parameters. The parameter $\alpha$ is called the index of stability, $\sigma$ the scale parameter, $\beta$ the skewness parameter and $\mu$ is the location parameter. Stable variable $X$ is denoted by $X \sim S_{\alpha}(\sigma, \beta, \mu)$, whereas symmetric $\alpha$-stable random variable is denoted by $X \sim S \alpha S$. In the latter case $\beta=\mu=0$. When $\alpha=2$, the distribution reduces to Gaussian. For the Gaussian distribution $\beta$ is irrelevant, and it is characterized by standard deviation and mean.

It is known that for general stable distributions, although they are continuous, there is no elementary form of probability density function. Computational formulas for stable densities and distribution functions are given in 63 . The Fox function representation for the stable distributions is exhibited in [68] and stable densities in terms of the incomplete hypergeometric function are expressed in [35. The only stable distributions with elementary probability density functions are: the Gaussian distribution, namely $X \sim S_{2}(\sigma, 0, \mu)=$ $N\left(\mu, 2 \sigma^{2}\right)$, the Cauchy distribution, namely $X \sim S_{1}(\sigma, 0, \mu)$, and the Lèvy distribution, namely $X \sim S_{\frac{1}{2}}(\sigma, 1, \mu)$.

The tails of non-Gaussian stable distributions decrease like a power function: $|x|^{-\alpha}$. Due to this reason, they appear naturally in the description of random processes with large outliers. The rate of decay depends mainly on the parameter $\alpha$. The smaller the $\alpha$, the slower the decay and the heavier the tails. Consequently, for a stable random variable $X$ with index $\alpha<2$ one has $\left\langle|X|^{\delta}\right\rangle=\infty$ for any $\delta \geq \alpha$ and $<|X|^{\delta}><\infty$ for $0<\delta<\alpha$. The comprehensive theory of $\alpha$-stable distributions is presented in [38].

There are at least three standard procedures for estimating stable law parameters: (i) the maximum likelihood method based on numerical approximation of the stable likelihood function; (ii) the quantile method using tabulated quantiles of stable laws; and (iii) the method using regression on the sample characteristic function. The regression method is considered to be both fast and accurate $([79])$. 
If the analyzed data come from FLSM or ARFIMA with Lévy stable noise, one can apply the computer test from [75] to estimate the stability index $\alpha$ of the data. The test applies the concept of surrogate data ([21]), which refers to data that preserve certain linear statistic properties of the experimental time series, without the deterministic component. If the data come from FLSM or ARFIMA, then we should observe a change to $1 / \alpha$ in the self-similarity estimator values for FLSM and aggregate ARFIMA.

\section{Algorithm 6. Computer test to estimate the stability index ([75])}

(i) Obtain the surrogate data, e.g. by random shuffling of the original data positions.

(ii) Estimate the self-similarity index $H$, e.g. via the p-variation algorithm (see Appendix $F$ ). The resulting $\alpha=1 / H$.

The test can be also used to distinguish between diffusion (BM and LSM) and fractional (anomalous) diffusion (FBM and FLSM) models.

To test whether a data follow a Gaussian distribution it is enough to apply one of the standard tests implemented in many many mathematical packages, like, e.g. Jarque-Berra or Kolmogorov-Smirnov ([17]). For the non-Gaussian stable distribution one can apply strict testing procedure for an arbitrary distribution or to employ specific properties of the stable law. We concentrate here on the latter case. One way to test for stability is to check the distinctive property of stable random variables, namely summability, see, e.g., [4]. The second array of tests checks the power-law behaviour of the underlying data. In this context an issue arises of recognizing stable distributions with the stability index close to 2 . In this case, if the sample is not long enough, the shape of empirical PDF is close to a Gaussian ([17]), and both log-log scale analysis and standard estimators of the power-law exponent estimators like Hill give overestimated value of $\alpha$ for the number of observation less than $10^{6}$ ([78]). In applications, the number of observations is often less.

The problem of recognizing $\alpha$-stable distribution with $\alpha$ close to 2 from experimental data when the sample size of available data is not large, was addressed in [17]. Following [17] we introduce a testing procedure combining a simple visual test based on empirical fourth moment, and the AndersonDarling (AD) and Jarque-Bera (JB) statistical tests. Namely, we calculate the empirical cumulative fourth moment (ECFM) of the simulated data sets, which for a sample of observations $\left\{x_{1}, \ldots, x_{n}\right\}$ is defined as follows:

$$
C(k)=\frac{1}{k} \sum_{i=1}^{k}\left(x_{i}-\bar{x}\right)^{4}, k=1,2, \ldots, n,
$$

where $\bar{x}$ is the mean of the random sample. 
Formula (E.1) can be calculated for any sample obtained from an arbitrary probability distribution. For a fixed $k$ it forms a random variable. For distributions with finite fourth moment (e.g. Gaussian), the ECFM, as a function of $k$, converges to a constant, whereas for distributions with infinite fourth moment (e.g. stable with $\alpha<2$ ) it diverges to infinity. The latter, for a finite sample, can be observed as an irregular chaotic behaviour. The resulting procedure is presented in the following algorithm.

Algorithm 7. Recognizing $\alpha$-stable distribution with $\alpha$ close to 2 ([17])

(i) Calculate the ECFM for the sample.

(ii) If the ECFM tends to a constant, we check for the Gaussian distribution by using the JB test.

(a) If its p-value exceeds the confidence level (usually 5\%), then we can assume the underlying distribution of time series is Gaussian. In this case we estimate its parameters by using the standard maximum likelihood estimation method.

(b) If the JB test shows the data cannot be modeled by a Gaussian distribution, then we test them for the stable distribution.

1. If the $A D$ test gives the p-value that exceeds the confidence level, then we can assume the time series can be described by the $\alpha$ stable distribution. In this case we estimate its parameters via the regression approach.

2. If the stability is rejected, other distributions have to be take into considerations.

(iii) If the ECFM exhibits chaotic-like behaviour, then we test for the stable distribution by means of AD test.

(a) If the p-value is greater than the confidence level, then we can assume the data follow the stable law. In this case we estimate its parameters via the regression approach.

(b) If the stability is rejected, other distributions have to be take into considerations.

\section{Appendix F. Self-similarity index estimator based on sample $p$-variation}

Let us now discuss the idea of $p$-variation, $p>0$. The concept of $p$-variation generalizes the well-known notions of total or quadratic variations, which have found applications in various areas of physics, mathematics and engineering $([57,76])$. Let $X(t)$ be a stochastic process analyzed on the time interval $[0, T]$. Then, the $p$-variation of $X(t)$ is defined as the limit of sum of increments of 
$X(t)$ taken to the $p$-th power over all partitions $P$ of the interval $[0, T]$, when the mesh of the partitions goes to zero. When $p=1$ it reduces to the total variation, whereas $p=2$ leads to the notion of quadratic variation.

In practice, one calculates sample $p$-variation ([15]) taking differences between every $m$-th element of the data.

Let $\left\{X_{i}, i=0, \ldots, N\right\}$ be a sample of length $N+1$. Sample $p$-variation $V_{m}^{(p)}$ for lag $m$ is defined as

$$
V_{m}^{(p)}=\sum_{k=0}^{N / m-1}\left|X_{(k+1) m}-X_{k m}\right|^{p} .
$$

The following fact describes the behaviour of the $p$-variation for the partial sum process of the ARFIMA time series. Let $\left\{Y_{i}, i=0, \ldots, N\right\}$ be a $\operatorname{ARFIMA}(p, d, q)$ time series with $\alpha$-stable noise and $1<\alpha \leq 2$. We define its partial sum process $\left\{X_{k}=\sum_{i=1}^{k} Y_{i}, k=0, \ldots, N\right\}$. Then for large $N / m$ : if $\alpha=2$, then $V_{m}^{(p)} \sim m^{H p-1}$; if $1<\alpha<2$, then $V_{m}^{(p)} \sim m^{H p-1}$ for $d>0$ and $V_{m}^{(p)} \sim m^{p(H-1 / \alpha)}$ for $d<0$. The symbol $\sim$ denotes similarity in distribution and $H=d+1 / \alpha$.

Since the normalized partial sum of the ARFIMA process converges to a FLSM with $H=d+1 / \alpha([74])$, we obtain the following fact. Let $\left\{X_{i}, i=\right.$ $0, \ldots, N\}$ be a FLSM with $0<H<1$ and $1<\alpha \leq 2$. Then for large $N / m$ : if $\alpha=2$, then $V_{m}^{(p)} \sim m^{H p-1}$; if $1<\alpha<2$, then $V_{m}^{(p)} \sim m^{H p-1}$ for $H>1 / \alpha$ and $V_{m}^{(p)} \sim m^{p(H-1 / \alpha)}$ for $H<1 / \alpha$. The symbol $\sim$ denotes similarity in distribution.

This implies that, in the case of Gaussian ARFIMA or FBM for $p>1 / H$, sample $p$-variation is a strictly increasing function of $m$ (it tends to zero as $m$ gets smaller), whereas for $p<1 / H$ it is a strictly decreasing function of $m$ (it diverges to infinity when $m$ gets smaller). For a stable non-Gaussian ARFIMA or FLSM the situation differs from the Gaussian case and depends on whether $d$ is positive or negative. It appears that the sample $p$-variation is always a decreasing function with respect to $m$ when $d<0$. If $d>0$, the situation is the same as in the Gaussian case: if $p>1 / H$, then sample $p$-variation is an increasing function of $m$, if $p<1 / H$ it is a decreasing function of $m$.

Now, we introduce a method of estimation of the self-similarity index $H$ based on sample $p$-variation for the ARFIMA process and fractional stable motion. For $d>0$ the method applies to both Gaussian $(\alpha=2)$ and nonGaussian $(\alpha<2)$ cases. For $d<0$ it is defined only for the Gaussian case. The idea of the method is to find $p=1 / H$ for which $V_{m}^{(p)}$ as a function of $m$ changes its monotonic behaviour, i.e. becomes constant. To this end, as a simple tool, we propose to calculate difference between $V_{m}^{(p)}$ for the smallest $m$, namely $m=1$, and $V_{m}^{(p)}$ for a larger $m$ (but not too large to make sure 
$N / m$ is sufficiently high), e.g. $m=8$. This is done for different $p$ 's. The smallest distance defines the value of the estimator $\hat{p}$ and consequently $\hat{H}$. The procedure can be summarized as follows.

Algorithm 8. Estimation of the self-similarity index based on sample p-variation ([7])

(i) Calculate $V_{m}^{(p)}$ for $p=1 . /(0.01: 0.01: 0.99)$ and $m=1$ and 8 .

(ii) Find $\hat{p}$ that minimizes $\left(V_{8}^{(p)}-V_{1}^{(p)}\right)^{2}$.

(iii) The estimated value $\hat{H}=1 / \hat{p}$.

The introduced $p$-variation estimator seems to be consistent and works remarkably well for the FBM case. It is worth-mentioning that the variance of the estimator is very low in comparison to other methods of estimation of the self-similarity index ([7]).

Finally, we note that the idea of $p$-variation can be used to distinguish between two mechanisms leading to anomalous diffusion, namely FBM and CTRW. In [57] a simple test, based on a realization of the unknown process, was introduced and applied to the data of Golding and Cox ([30]).

\section{References}

[1] Barkai E., Metzler R., Klafter J., From continuous time random walks to the fractional Fokker-Planck equation, Phys. Rev. E 61 (2000), 132-138.

[2] Barndorff-Nielsen O.E., Normal//inverse Gaussian Processes and the Modelling of Stock Returns, Research Report 300, Department of Theoretical Statistics, University of Aarhus, 1995.

[3] Beran J., Statistics for Long-Memory Processes, Chapman and Hall, New York, 1994.

[4] Brcich R.F., Iskander D.R., Zoubir A.M., The stability test for symmetric alpha-stable distributions, IEEE Trans. Signal Process. 53 (2005), 977-986.

[5] Brockwell P.J., Davis R.A., Introduction to Time Series and Forecasting, SpringerVerlag, New York, 2002.

[6] Burnecki K., FARIMA processes with application to biophysical data, J. Stat. Mech. 2012, P05015, 18 pp.

[7] Burnecki K., Identification, Validation and Prediction of Fractional Dynamical Systems, Wroclaw University of Technology Press, Wrocław, 2012.

[8] Burnecki K., Gajda J., Sikora G., Stability and lack of memory of the returns of the Hang Seng index, Physica A 390 (2011), 3136-3146.

[9] Burnecki K., Kepten E., Garini Y., Sikora G., Weron A., Estimating the anomalous diffusion exponent for single particle tracking data with measurement errors - An alternative approach, Sci. Reports 5 (2015), 11306, 11 pp.

[10] Burnecki K., Kepten E., Janczura J., Bronshtein I., Garini Y., Weron A., Universal algorithm for identification of fractional Brownian motion. A case of telomere subdiffusion, Biophys. J. 103 (2012), 1839-1847. 
[11] Burnecki K., Magdziarz M., Weron A., Identification and validation of fractional subdiffusion dynamics, in: Klafter J., Lim S.C., Metzler R. (eds.), Fractional Dynamics. Recent Advances, World Scientific, Singapore, 2012, pp. 331-351.

[12] Burnecki K., Muszkieta M., Sikora G., Weron A., Statistical modelling of subdiffusive dynamics in the cytoplasm of living cells: A FARIMA approach, EPL 98 (2012), 10004, $6 \mathrm{pp}$.

[13] Burnecki K., Sikora G., Estimation of FARIMA parameters in the case of negative memory and stable noise, IEEE Trans. Signal Process. $61(2013), \overline{28} 2 \overline{25-2835}$.

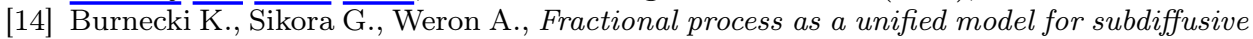
dynamics in experimental data, Phys. Rev. E 86 (2012), 041912, 8 pp.

[15] Burnecki K., Weron A., Fractional Lévy stable motion can model subdiffusive dynamics, Phys. Rev. E 82 (2010), 021130, 8 pp.

[16] Burnecki K., Weron A., Algorithms for testing of fractional dynamics: a practical guide to ARFIMA modelling, J. Stat. Mech. 2014, P10036, 26 p.

[17] Burnecki K., Wyłomańska A., Beletskii A., Gonchar V., Chechkin A., Recognition of stable distribution with Lévy index a close to 2, Phys. Rev. E 85 (2012), 056711, 8 pp.

[18] Caccia D.C., Percival D., Cannon M.J., Raymond G., Bassingthwaighte J.B., Analyzing exact fractal time series: evaluating dispersional analysis and rescaled range methods, Physica A 246 (1997), 609-632.

[19] Cambanis S., Podgórski K., Weron A., Chaotic behavior of infinitely divisible processes, Studia Math. 115 (1995), 109-127.

[20] Chan T.F., Vese L.A., Active contours without edges, IEEE Trans. Image Process. 10 (2001), 266-277.

[21] Chang T., Sauer T., Schiff S.J., Tests for nonlinearity in short stationary time series, Chaos 5 (1995), 118-126.

[22] Clark S., The dark side of the Sun, Nature 441 (2006), 402-404.

[23] Crato N., Rothman P., Fractional integration analysis of long-run behavior for US macroeconomic time series, Econom. Lett. 45 (1994), 287-291.

[24] Davies R.B., Harte D.S., Tests for Hurst effect, Biometrika 74 (1987), 95-101.

[25] Fleck L., Kowarzyk H., Steinhaus H., La distribution des leucocytes dans les préparations du sang, J. Suisse de Médecine 77 (1947), 1283.

[26] Fouskitakis G.N., Fassois S.D., Pseudolinear estimation of fractionally integrated ARMA (ARFIMA) models with automotive application, IEEE Trans. Signal Process. 47 (1999), 3365-3380.

[27] Fuliński A., Communication: How to generate and measure anomalous diffusion in simple systems, J. Chem. Phys. 138 (2013), 021101, 4 pp.

[28] Gajda J., Sikora G., Wyłomańska A., Regime variance testing - a quantile approach, Acta Phys. Pol. B 44 (2013), 1015-1035.

[29] Gil-Alana L., A fractionally integrated model for the Spanish real GDP, Econ. Bull. 3 (2004), 1-6.

[30] Golding I., Cox E.C., Physical nature of bacterial cytoplasm, Phys. Rev. Lett. 96 (2006), 098102, 4 pp.

[31] Granger C.W.J., Joyeux R., An introduction to long-memory time series models and fractional differencing, J. Time Ser. Anal. 1 (1980), 15-29.

[32] Guigas G., Kalla C., Weiss M., Probing the nanoscale viscoelasticity of intracellular fluids in living cells, Biophys. J. 93 (2007), 316-323.

[33] Havlin S., Ben-Avraham D., Diffusion in disordered media, Adv. Phys. 36 (1987), 695-798.

[34] Hellmann M., Klafter J., Heermann D.W., Weiss M., Challenges in determining anomalous diffusion in crowded fluids, J. Phys.: Condens. Matter 23 (2011), 234113, $7 \mathrm{pp}$.

[35] Hoffmann-Jørgensen J., Stable densities, Theory Probab. Appl. 38 (1993), 350-355.

[36] Höfling F., Franosch T., Anomalous transport in the crowded world of biological cells, Rep. Prog. Phys. 76 (2013), 046602, 50 pp.

[37] Hosking J.R.M., Fractional differencing, Biometrika 68 (1981), 165-176. 
[38] Janicki A., Weron A., Simulation and Chaotic Behavior of $\alpha$-stable Stochastic Processes, Marcel Dekker Inc., New York, 1994.

[39] Kehr K.W., Kutner R., Random walk on a random walk, Physica A 110 (1982), 535-549.

[40] Kokoszka P.S., Prediction of infinite variance fractional ARIMA, Probab. Math. Statist. 16 (1996), 65-83.

[41] Kokoszka P.S., Taqqu M.S., Fractional ARIMA with stable innovations, Stochastic Process. Appl. 60 (1995), 19-47.

[42] Kokoszka P.S., Taqqu M.S., Parameter estimation for infinite variance fractional ARIMA, Ann. Statist. 24 (1996), 1880-1913.

[43] Kolmogorov A.N., Wienersche Spiralen und einige andere interessante Kurven im Hilbertschen Raum, C. R. (Doklady) Acad. Sci. URSS (N.S.) 26 (1940), 115-118.

[44] Kou S.C., Xie X.S., Generalized Langevin equation with fractional Gaussian noise: subdiffusion within a single protein molecule, Phys. Rev. Lett. 93 (2004), 180603, 4 pp.

[45] Kowarzyk H., Steinhaus H., Szymaniec S., Arrangement of chromosomes in human cells. I. Associations in metaphase figures, Bull. Acad. Polon. Sci., Ser. Sci. Biol. 13 (1965), 321-326.

[46] Kowarzyk H., Steinhaus H., Szymaniec S., Arrangement of chromosomes in human cells. 3. Distribution of centromeres and orientation of chromosomes in the metaphase, Bull. Acad. Polon. Sci., Ser. Sci. Biol. 14 (1966), 541-544.

[47] Lagarias J.C., Reeds J.A., Wright M.H., Wright P.E., Convergence properties of the Nelder-Mead simplex method in low dimensions, SIAM J. Optim. 9 (1998), 112-147.

[48] Lanoiselée Y., Grebenkov D.S., Revealing nonergodic dynamics in living cells from a single particle trajectory, Phys. Rev. E 93 (2016), 052146, 17 pp.

[49] Lasota A., Mackey M.C., Chaos, Fractals, and Noise. Stochastic Aspects of Dynamics, Springer-Verlag, New York, 1994.

[50] Lasota A., Mackey M.C., Ważewska-Czyżewska M., Minimizing therapeutically induced anemia, J. Math. Biol. 13 (1981/82), 149-158.

[51] Lim S.C., Muniandy S.V., Self-similar Gaussian processes for modeling anomalous diffusion, Phys. Rev. E 66 (2002), 021114, 14 pp.

[52] Ljung G.M., Box G.E.P., On a measure of lack of fit in time series models, Biometrika 65 (1978), 297-303.

[53] Loch H., Janczura A., Weron A., Ergodicity testing using an analytical formula for a dynamical functional of alpha-stable autoregressive fractionally integrated moving average processes, Phys. Rev. E 93 (2016), 043317, 10 pp.

[54] Loch-Olszewska H., Sikora G., Janczura J., Weron A., Identifying ergodicity breaking for fractional anomalous diffusion: Criteria for minimal trajectory length, Phys. Rev. E 94 (2016), 052136, 8 pp.

[55] Magdziarz M., Weron A., Fractional Langevin equation with $\alpha$-stable noise. A link to fractional ARIMA time series, Studia Math. 181 (2007), 47-60.

[56] Magdziarz M., Weron A., Anomalous diffusion: Testing ergodicity breaking in experimental data, Phys. Rev. E 84 (2011), 051138, 5 pp.

[57] Magdziarz M., Weron A., Burnecki K., Klafter J., Fractional Brownian motion versus the continuous-time random walk: a simple test for subdiffusive dynamics, Phys. Rev. Lett. 103 (2009), 180602, 4 pp.

[58] Mandelbrot B.B., Wallis J.R., Noah, Joseph, and operational hydrology, Water Resour. Res. 4 (1968), 909-918.

[59] Mann M.E., Bradley R.S., Hughes M.K., Global-scale temperature patterns and climate forcing over the past six centuries, Nature 392 (1998), 779-787.

[60] Meroz Y., Sokolov I.M., Klafter J., Test for determining a subdiffusive model in ergodic systems from single trajectories, Phys. Rev. Lett. 110 (2013), 090601, 4 pp.

[61] Metzler R., Barkai E., Klafter J., Anomalous diffusion and relaxation close to thermal equilibrium: a fractional Fokker-Planck equation approach, Phys. Rev. Lett. 82 (1999), 3563-3567. 
[62] Metzler R., Klafter J., The random walk's guide to anomalous difusion: a fractional dynamics approach, Phys. Rep. 339 (2000), 1-77.

[63] Nolan J.P., Numerical calculation of stable densities and distribution functions, Commun. Statist.-Stochastic Models 13 (1997), 759-774.

[64] Samko S.G., Kilbas A.A., Marichev O.I., Fractional Integrals and Derivatives. Theory and Applications, Gordon and Breach Science Publishers, Amsterdam, 1993.

[65] Saxton M.J., Anomalous diffusion due to obstacles: a Monte Carlo study, Biophys. J. 66 (1994), 394-401.

[66] Saxton M.J., Anomalous subdiffusion in fluorescence photobleaching recovery: a Monte Carlo study, Biophys. J. 81 (2001), 2226-2240.

[67] Saxton M.J., Wanted: a positive control for anomalous subdiffusion, Biophys. J. 103 (2012), 2411-2422.

[68] Schneider W.R., Stable distributions: Fox functions representation and generalization, in: Albeverio S., Casati G., Merlini D. (eds.), Stochastic Processes in Classical and Quantum Systems, Springer, Berlin, 1986, pp. 497-511.

[69] Ślęzak J., Drobczyński D., Weron K., Masajada J., Moving average process underlying the holographic-optical-tweezers experiments, Appl. Opt. 53(2014), B254-B258.

[70] Sokolov I.M., Models of anomalous diffusion in crowded environments, Soft Matter 8 (2012), 9043-9052.

[71] Stanislavsky A., Memory effects and macroscopic manifestation of randomness, Phys. Rev. E 61 (2000), 4752-4759.

[72] Stanislavsky A.A., Burnecki K., Magdziarz M., Weron A., Weron K., FARIMA modeling of solar flare activity from empirical time series of soft X-ray solar emission, Astrophys. J. 693 (2009), 1877-1882.

[73] Stanislavsky A., Weron K., Weron A., Anomalous diffusion with transient subordinators: a link to compound relaxation laws, J. Chem. Phys. 140 (2014), 054113, 7 pp.

[74] Stoev S., Taqqu M.S., Simulation methods for linear fractional stable motion and FARIMA using the fast Fourier transform, Fractals $12 \overline{(2004), 95}-\overline{121 .}$

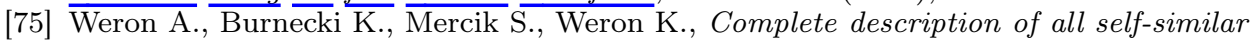
models driven by Lévy stable noise, Phys. Rev. E 71 (2005), 016113, 10 pp.

[76] Weron A., Magdziarz M., Anomalous diffusion and semimartingales, EPL 86 (2009), 60010, 6 pp.

[77] Weron A., Weron R., Computer simulation of Lévy $\alpha$-stable variables and processes, in: Garbaczewski P., Wolf M., Weron A. (eds.), Chaos - the Interplay Between Stochastic and Deterministic Behaviour, Springer, Berlin, 1995, pp. 379-392.

[78] Weron R., Levy-stable distributions revisited: tail index $>2$ does not exclude the Levystable regime, Int. J. Mod. Phys. C 12 (2001), 209-223.

[79] Weron R., Computationally intensive value at risk calculations, in: Gentle J.E., Härdle W., Mori Y. (eds.), Handbook of Computational Statistics. Concepts and Methods, Springer, Berlin, 2004, pp. 911-950.

[80] Wigner E.P., The unreasonable effectiveness of mathematics in the natural sciences, Comm. Pure Appl. Math. 13 (1960), 1-14.

Hugo Steinhaus Center

Faculty of Pure and Aplied Mathematics

Wroclaw University of Science and Technology

WYSPIANSKIEGO 27

50-370 Wroclaw

POLAND

e-mail: aleksander.weron@pwr.edu.pl 